\title{
Second Order Symmetries of the Conformal Laplacian
}

\author{
Jean-Philippe MICHEL ${ }^{\dagger}$, Fabian RADOUX ${ }^{\dagger}$ and Josef ŠILHAN ${ }^{\ddagger}$ \\ $\dagger$ Department of Mathematics of the University of Liège, \\ Grande Traverse 12, 4000 Liège, Belgium \\ E-mail: jean-philippe.michel@ulg.ac.be,fabian.radoux@ulg.ac.be \\ URL: http://www.geodiff.ulg.ac.be/recherche/fradoux/, \\ http://www.geodiff.ulg.ac.be/recherche/michel/ \\ $\ddagger$ Department of Algebra and Geometry of the Masaryk University in Brno, \\ Janàčkovo nàm. 2a, 662 95 Brno, Czech Republic \\ E-mail: silhan@math.muni.cz \\ URL: http://www.math.muni.cz/ silhan/
}

Received October 25, 2013, in final form February 05, 2014; Published online February 14, 2014 http://dx.doi.org/10.3842/SIGMA.2014.016

\begin{abstract}
Let $(M, \mathrm{~g})$ be an arbitrary pseudo-Riemannian manifold of dimension at least 3. We determine the form of all the conformal symmetries of the conformal (or Yamabe) Laplacian on $(M, \mathrm{~g})$, which are given by differential operators of second order. They are constructed from conformal Killing 2-tensors satisfying a natural and conformally invariant condition. As a consequence, we get also the classification of the second order symmetries of the conformal Laplacian. Our results generalize the ones of Eastwood and Carter, which hold on conformally flat and Einstein manifolds respectively. We illustrate our results on two families of examples in dimension three.
\end{abstract}

Key words: Laplacian; quantization; conformal geometry; separation of variables

2010 Mathematics Subject Classification: 58J10; 53A30; 70S10; 53D20; 53D55

\section{Introduction}

We work over a pseudo-Riemannian manifold $(M, \mathrm{~g})$ of dimension $n \geq 3$, with Levi-Civita connection $\nabla$ and scalar curvature Sc. Our main result is the classification of all the differential operators $D_{1}$ of second order such that the relation

$$
\Delta_{Y} D_{1}=D_{2} \Delta_{Y}
$$

holds for some differential operator $D_{2}$, where $\Delta_{Y}:=\nabla_{a} \mathrm{~g}^{a b} \nabla_{b}-\frac{n-2}{4(n-1)}$ Sc is the Yamabe Laplacian. Such operators $D_{1}$ are called conformal symmetries of order 2 of $\Delta_{Y}$. They preserve the kernel of $\Delta_{Y}$, i.e. the solution space of the equation $\Delta_{Y} \psi=0, \psi \in \mathcal{C}^{\infty}(M)$. Under a conformal change of metric, $\hat{\mathrm{g}}=e^{2 \Upsilon \mathrm{g}}, \Upsilon \in \mathcal{C}^{\infty}(M)$, the Yamabe Laplacian transforms as

$$
\widehat{\Delta_{Y}}=e^{-\frac{n+2}{2} \Upsilon} \circ \Delta_{Y} \circ e^{\frac{n-2}{2} \Upsilon},
$$

so that each conformal symmetry $D_{1}$ of $\Delta_{Y}$ gives rise to one of $\widehat{\Delta_{Y}}$ given by

$$
\widehat{D_{1}}=e^{-\frac{n-2}{2} \Upsilon} \circ D_{1} \circ e^{\frac{n-2}{2} \Upsilon} .
$$

This emphasizes the conformal nature of the problem and justify our choice of the Yamabe Laplacian, rather than the more usual Laplace-Beltrami one, $\Delta:=\nabla_{a} \mathrm{~g}^{a b} \nabla_{b}$. Over flat pseudoEuclidean space, the classification of conformal symmetries up to second order is due to Boyer, 
Kalnins and Miller [7], who use it to study the $R$-separation of variables of the Laplace equation $\Delta \Psi=0$. More generally, Kalnins and Miller provide an intrinsic characterization for $R$-separation of this equation on $(M, \mathrm{~g})$ in terms of second order conformal symmetries [19]. Thus, classifying those symmetries happens to be a basic problem in the theory of separation of variables. A new input into the quest of conformal symmetries has been given by the work of Eastwood [15]. He classified indeed the conformal symmetries of any order over the conformally flat space and exhibited their interesting algebraic structure. This leads to a number of subsequent works, dealing with other invariant operators [16, 18, 31].

Using principal symbol maps, one can extract two informations from the equation (1.1): the operators $D_{1}$ and $D_{2}$ have the same principal symbol and the latter is a conformal Killing 2tensor, i.e. a constant of motion of the geodesic flow, restricted to the null cone. One looks then for a right inverse to the principal symbol maps, called a quantization map, which associates with each conformal Killing tensor a conformal symmetry of $\Delta_{Y}$. For Killing vector fields this is trivial. If $K$ is a 2-tensor, Carter proves that if the minimal prescription

$$
K \mapsto \nabla_{a} K^{a b} \nabla_{b}
$$

satisfies $\left[\Delta_{Y}, \nabla_{a} K^{a b} \nabla_{b}\right]=0$, then $K$ is Killing. Moreover, he shows that if $(M, \mathrm{~g})$ is Einstein, i.e. if Ric $=\frac{1}{n}$ Scg with Ric the Ricci tensor, the fact that $K$ is Killing is sufficient to ensure that the minimal prescription above is a symmetry of $\Delta_{Y}$ (for application to the separation of variables, see [3]). Besides, in [15], Eastwood defines conformally invariant operators on an arbitrary pseudo-Riemannian manifold, which coincide with the conformal symmetries of $\Delta_{Y}$ on the flat space. These operators are given by means of the natural and conformally invariant quantization $\mathcal{Q}_{\lambda_{0}, \lambda_{0}}$ (where $\lambda_{0}=\frac{n-2}{2 n}$ ), developed in [9, 24, 29, 30]. Explicitly, if $X$ is a vector field and $K$ a symmetric trace-less 2 -tensor, $\mathcal{Q}_{\lambda_{0}, \lambda_{0}}(X)$ and $\mathcal{Q}_{\lambda_{0}, \lambda_{0}}(K)$ are differential operators acting between $\lambda_{0}$-densities defined in the following way:

$$
\begin{aligned}
& \mathcal{Q}_{\lambda_{0}, \lambda_{0}}(X)=X^{a} \nabla_{a}+\frac{n-2}{2 n}\left(\nabla_{a} X^{a}\right), \\
& \mathcal{Q}_{\lambda_{0}, \lambda_{0}}(K)=K^{a b} \nabla_{a} \nabla_{b}+\frac{n}{n+2}\left(\left(\nabla_{a} K^{a b}\right) \nabla_{b}+\frac{n-2}{4(n+1)}\left(\nabla_{a} \nabla_{b} K^{a b}\right)\right)-\frac{n+2}{4(n+1)} \operatorname{Ric}_{a b} K^{a b} .
\end{aligned}
$$

In the conformally flat case, all the conformal symmetries of second order are of the type $\mathcal{Q}_{\lambda_{0}, \lambda_{0}}(K+X+c)$, where $c \in \mathbb{R}, X$ is a conformal Killing vector field and $K$ is a conformal Killing 2-tensor. Thanks to the conformal covariance of $\Delta_{Y}$ one can show that $\mathcal{Q}_{\lambda_{0}, \lambda_{0}}(X)$ is still a conformal symmetry of $\Delta_{Y}$ on an arbitrary pseudo-Riemannian manifold, if $X$ is a conformal Killing vector field. However, as pointed out by Eastwood in [15], it is unclear whether $\mathcal{Q}_{\lambda_{0}, \lambda_{0}}(K)$ is a conformal symmetry when $K$ is a conformal Killing 2-tensor.

Our strategy relies on the properties of the quantization map $\mathcal{Q}_{\lambda_{0}, \lambda_{0}}$ and on the classification of natural and conformally invariant operators acting on prescribed subspaces of symbols. This method has been developed first on conformally flat manifolds, in [26]. In that case, the map $\mathcal{Q}_{\lambda_{0}, \lambda_{0}}$ is a conformally equivariant quantization [12], and the author proved that it is precisely the bijective map between conformal Killing tensors and conformal symmetries of $\Delta_{Y}$, discovered by Eastwood. The description of conformal symmetries on arbitrary pseudo-Riemannian manifolds is more involved, even at order 2. Namely, there exists a conformal symmetry with principal symbol $K$ if and only if $K$ is a conformal Killing tensor and $\operatorname{Obs}(K)^{b}$ is an exact one-form. Here, Obs is a natural and conformally invariant operator which reads, in abstract index notation, as

$$
\operatorname{Obs}(K)^{a}=\frac{2(n-2)}{3(n+1)}\left(\mathrm{C}^{r}{ }_{s t}^{a} \nabla_{r}-3 \mathrm{~A}_{s t}{ }^{a}\right) K^{s t},
$$


where $\mathrm{C}$ denotes the Weyl tensor and $\mathrm{A}$ the Cotton-York tensor. If $\mathbf{O b s}(K)^{\mathrm{b}}$ is equal to the exact one-form $-2 d f$, with $f \in \mathcal{C}^{\infty}(M)$, then the operators

$$
\mathcal{Q}_{\lambda_{0}, \lambda_{0}}(K+X+c)+f
$$

are conformal symmetries of $\Delta_{Y}$ for all conformal Killing vector field $X$ and constant $c \in \mathbb{R}$. As a consequence, $\mathcal{Q}_{\lambda_{0}, \lambda_{0}}(K)$ is a conformal symmetry of $\Delta_{Y}$ if and only if $\operatorname{Obs}(K)=0$.

We illustrate our results on two examples in dimension three. In the first one, the space $\mathbb{R}^{3}$ is endowed with the most general Riemannian metric admitting a Killing 2-tensor $K$, which is diagonal in orthogonal coordinates [28]. Then, $\operatorname{Obs}(K)^{b}$ is a non-trivial exact 1-form and, up to our knowledge, the symmetry of $\Delta_{Y}$ that we obtain is new. In the second one, we consider a conformal Stäckel metric g on $\mathbb{R}^{3}$ with one ignorable coordinate. Such a metric admits an irreducible conformal Killing tensor $K$. Using the generic form of $\mathrm{g}$ and $K$ given in the reference [8], we obtain that $\operatorname{Obs}(K)^{b}$ is a non-exact 1-form in general. This means there are no conformal symmetries of $\Delta_{Y}$ with principal symbol $K$ in general.

We detail now the content of the paper.

In Section 2, we introduce the basic spaces: the one of tensor densities $\mathcal{F}_{\lambda}(M)$ of weight $\lambda \in \mathbb{R}$, the one of differential operators $\mathcal{D}_{\lambda, \mu}(M)$ acting between $\lambda$ - and $\mu$-densities, the one of symbols $\mathcal{S}_{\delta}(M)$ with $\delta=\mu-\lambda$. Then, we define the Yamabe Laplacian $\Delta_{Y}$ as an element of $\mathcal{D}_{\lambda_{0}, \mu_{0}}(M)$, with $\lambda_{0}=\frac{n-2}{2 n}$ and $\mu_{0}=\frac{n+2}{2 n}$, so that it becomes a conformally invariant operator. Finally we introduce our main tool, namely the natural and conformally invariant quantization

$$
\mathcal{Q}_{\lambda, \mu}: \mathcal{S}_{\mu-\lambda}(M) \rightarrow \mathcal{D}_{\lambda, \mu}(M),
$$

and we provide explicit formulas for it.

In Section 3, we classify the natural and conformally invariant operators between some subspaces of symbols. Among the operators we obtain (and which are crucial for understanding of 2nd order symmetries), one of them, $\mathbf{G}$, is classical, whereas another one, Obs, acting on symbols of second degree, is new and admits no counterpart on flat space. We obtain also an analogous classification for higher order trace-free symbols where the situation is much more complicated. Note that the discovered operators act between source and target spaces of wellknown conformally invariant operators, which appear in the generalized BGG sequence [10]. It would be interesting to understand better the relations between all these conformal operators.

In Section 4 lies our main result. After defining the spaces of conformal symmetries and of conformal Killing tensors, we prove that, on symbols $K$ of degree 2, we have

$$
\left(\mathcal{Q}_{\lambda_{0}, \mu_{0}}\right)^{-1}\left(\Delta_{Y} \mathcal{Q}_{\lambda_{0}, \lambda_{0}}(K)-\mathcal{Q}_{\mu_{0}, \mu_{0}}(K) \Delta_{Y}\right)=2 \mathbf{G}(K)+\operatorname{Obs}(K) .
$$

The kernel of $\mathbf{G}$ is precisely the space of conformal Killing tensors, whereas $\operatorname{Obs}(K)$ is the obstruction for a conformal Killing 2-tensor to provide a conformal symmetry of the form $\mathcal{Q}_{\lambda_{0}, \lambda_{0}}(K)$. The full description of conformal symmetries of 2 nd order of $\Delta_{Y}$ easily follows. Using that $\mathcal{Q}_{\lambda_{0}, \lambda_{0}}(K)=\mathcal{Q}_{\mu_{0}, \mu_{0}}(K)$ for Killing 2-tensors, we deduce also the classification of second order symmetries of $\Delta_{Y}$, which satisfy by definition $\left[\Delta_{Y}, D_{1}\right]=0$.

In Section 5, we provide two examples illustrating our main result. In the first one, the Killing tensor $K$ is such that $\operatorname{Obs}(K)^{b}$ is a non-vanishing but exact one-form. In the second example, we provide several conformal Killing tensors $K$ such that $\operatorname{Obs}(K)^{b}$ is a non-exact one-form. Hence, there is no conformal symmetry with such $K$ as principal symbols.

\section{Conformal geometry, differential operators, and their symbols}

Throughout this paper, we employ the abstract index notation from [27]. That is, on a smooth manifold $M, v^{a}$ denotes a section of the tangent bundle $T M, v_{a}$ a section of the cotangent bundle 
$T^{*} M$ and e.g. $v^{a b}{ }_{c}$ a section of $T M \otimes T M \otimes T^{*} M$. The letters $a, b, c, d$ and $r, s, t$ are reserved for abstract indices. Repetition of an abstract index in the covariant and contravariant position means contraction, e.g. $v_{b}^{a b}$ is a section of $T M$. In few places we use concrete indices attached to a coordinate system. This is always explicitly stated and we denote such indices by letters $i, j, k, l$ to avoid confusion with abstract indices. We always use the Einstein's summation convention for indices, except if stated otherwise.

\subsection{Basic objects}

Let $M$ be a $n$-dimensional smooth manifold. If $\lambda \in \mathbb{R}$, the vector bundle of $\lambda$-densities, $F_{\lambda}(M) \rightarrow M$, is a line bundle associated with $P^{1} M$, the linear frame bundle over $M$ :

$$
F_{\lambda}(M)=P^{1} M \times_{\rho} \mathbb{R}
$$

where the representation $\rho$ of the group $\operatorname{GL}(n, \mathbb{R})$ on $\mathbb{R}$ is given by

$$
\rho(A) e=|\operatorname{det} A|^{-\lambda} e, \quad \forall A \in \mathrm{GL}(n, \mathbb{R}), \quad \forall e \in \mathbb{R} .
$$

We denote by $\mathcal{F}_{\lambda}(M)$ the space of smooth sections of this bundle. Since $F_{\lambda}(M)$ is associated with $P^{1} M$, the space $\mathcal{F}_{\lambda}(M)$ is endowed with canonical actions of $\operatorname{Diff}(M)$ and $\operatorname{Vect}(M)$. If $\left(x^{1}, \ldots, x^{n}\right)$ is a coordinate system on $M$, we denote by $|D x|^{\lambda}$ the local $\lambda$-density equal to $[(\mathrm{Id}, 1)]$, where Id is the identity frame in the coordinates system $\left(x^{1}, \ldots, x^{n}\right)$.

Actually, a $\lambda$-density $\varphi$ at a point $x \in M$ can be viewed as a map on $\wedge^{n} T_{x} M$ with values in $\mathbb{R}$ such that

$$
\varphi\left(c X_{1} \wedge \cdots \wedge X_{n}\right)=|c|^{\lambda} \varphi\left(X_{1} \wedge \cdots \wedge X_{n}\right)
$$

for all $X_{1}, \ldots, X_{n} \in T_{x} M$ and $c \in \mathbb{R}$. The $\lambda$-density $|D x|^{\lambda}$ is then the $\lambda$-density equal to one on $\partial_{1} \wedge \cdots \wedge \partial_{n}$, where $\partial_{1}, \ldots, \partial_{n}$ denotes the canonical basis of $T_{x} M$ corresponding to the coordinate system $\left(x^{1}, \ldots, x^{n}\right)$.

If a $\lambda$-density $\varphi$ reads locally $f|D x|^{\lambda}$, where $f$ is a local function, then the Lie derivative of $\varphi$ in the direction of a vector field $X$ reads locally

$$
L_{X}^{\lambda} \varphi=\left(X . f+\lambda\left(\partial_{i} X^{i}\right) f\right)|D x|^{\lambda} .
$$

It is possible to define the multiplication of two densities. If $\varphi_{1}$ reads locally $f|D x|^{\lambda}$ and if $\varphi_{2}$ reads locally $g|D x|^{\delta}$, then $\varphi_{1} \varphi_{2}$ reads locally $f g|D x|^{\lambda+\delta}$.

On a pseudo-Riemannian manifold $(M, \mathrm{~g})$, it is possible to define in a natural way a $\lambda$-density. In a coordinate system, this $\lambda$-density reads

$$
\left|\operatorname{Vol}_{\mathrm{g}}\right|^{\lambda}=|\operatorname{det} \mathrm{g}|^{\frac{\lambda}{2}}|D x|^{\lambda}
$$

where $|\operatorname{det} g|$ denotes the absolute value of the determinant of the matrix representation of $g$ in the coordinate system.

We shall denote by $\mathcal{D}_{\lambda, \mu}(M)$ the space of differential operators from $\mathcal{F}_{\lambda}(M)$ to $\mathcal{F}_{\mu}(M)$. It is the space of linear maps between $\mathcal{F}_{\lambda}(M)$ and $\mathcal{F}_{\mu}(M)$ that read in trivialization charts as differential operators. The actions of $\operatorname{Vect}(M)$ and $\operatorname{Diff}(M)$ on $\mathcal{D}_{\lambda, \mu}(M)$ are induced by the actions on tensor densities: if $\mathcal{L}_{X} D$ denotes the Lie derivative of the differential operator $D$ in the direction of the vector field $X$, we have

$$
\begin{aligned}
& \mathcal{L}_{X} D=L_{X}^{\mu} \circ D-D \circ L_{X}^{\lambda}, \quad \forall D \in \mathcal{D}_{\lambda, \mu}(M) \quad \text { and } \quad \forall X \in \operatorname{Vect}(M) . \\
& \phi \cdot D=\phi \circ D \circ \phi^{-1}, \quad \forall D \in \mathcal{D}_{\lambda, \mu}(M) \quad \text { and } \quad \forall \phi \in \operatorname{Diff}(M) .
\end{aligned}
$$


The space $\mathcal{D}_{\lambda, \mu}(M)$ is filtered by the order of differential operators. We denote by $\mathcal{D}_{\lambda, \mu}^{k}(M)$ the space of differential operators of order $k$. It is well-known that this filtration is preserved by the action of local diffeomorphisms.

On a pseudo-Riemannian manifold $(M, \mathrm{~g})$, it is easy to build an isomorphism between $\mathcal{D}_{\lambda, \mu}(M)$ and $\mathcal{D}(M)$, the space of differential operators acting between functions. Indeed, thanks to the canonical densities built from $\left|\operatorname{Vol}_{\mathrm{g}}\right|$, all operators $D \in \mathcal{D}_{\lambda, \mu}(M)$ can be pulled-back on functions as follows

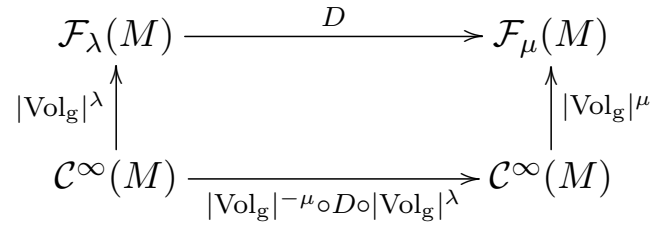

The space of symbols is the graded space associated with $\mathcal{D}_{\lambda, \mu}(M)$ : it is then equal to

$$
\operatorname{gr} \mathcal{D}_{\lambda, \mu}(M):=\bigoplus_{k=0}^{\infty} \mathcal{D}_{\lambda, \mu}^{k}(M) / \mathcal{D}_{\lambda, \mu}^{k-1}(M)
$$

The canonical projection $\sigma_{k}: \mathcal{D}_{\lambda, \mu}^{k}(M) \rightarrow \mathcal{D}_{\lambda, \mu}^{k}(M) / \mathcal{D}_{\lambda, \mu}^{k-1}(M)$ is called the principal symbol map. As the actions of $\operatorname{Diff}(M)$ and $\operatorname{Vect}(M)$ preserve the filtration of $\mathcal{D}_{\lambda, \mu}(M)$, they induce actions of $\operatorname{Diff}(M)$ and $\operatorname{Vect}(M)$ on the space of symbols.

Let $\delta=\mu-\lambda$ be the shift of weights. If the sum of the $k$-order terms of $D \in \mathcal{D}_{\lambda, \mu}^{k}$ in a coordinate system $\left(x^{1}, \ldots, x^{n}\right)$ reads

$$
D^{i_{1} \ldots i_{k}} \partial_{i_{1}} \cdots \partial_{i_{k}}
$$

and if $\left(x^{i}, p_{i}\right)$ is the coordinate system on $T^{*} M$ canonically associated with $\left(x^{1}, \ldots, x^{n}\right)$, then we get the following identification:

$$
\sigma_{k}(D) \quad \longleftrightarrow \quad D^{i_{1} \ldots i_{k}} p_{i_{1}} \cdots p_{i_{k}}
$$

Thus, the space of symbols of degree $k$ can be viewed as the space $\mathcal{S}_{\delta}^{k}(M):=\operatorname{Pol}^{k}\left(T^{*} M\right) \otimes_{\mathcal{C}^{\infty}(M)}$ $\mathcal{F}_{\delta}(M)$, where $\mathrm{Pol}^{k}\left(T^{*} M\right)$ denotes the space of real functions on $T^{*} M$ which are polynomial functions of degree $k$ in the fibered coordinates of $T^{*} M$. The algebra $\mathcal{S}(M):=\operatorname{Pol}\left(T^{*} M\right)$ is clearly isomorphic to the algebra $\Gamma(S T M)$ of symmetric tensors and depending on the context we will refer to its elements as symbols, functions on $T^{*} M$ or symmetric tensors on $M$.

Let us recall that, if $S_{1}, S_{2} \in \mathcal{S}(M)$, then the Poisson bracket of $S_{1}$ and $S_{2}$, denoted by $\left\{S_{1}, S_{2}\right\}$, is defined in a canonical coordinate system $\left(x^{i}, p_{i}\right)$ of $T^{*} M$ in the following way:

$$
\left\{S_{1}, S_{2}\right\}=\left(\partial_{p_{i}} S_{1}\right)\left(\partial_{x^{i}} S_{2}\right)-\left(\partial_{p_{i}} S_{2}\right)\left(\partial_{x^{i}} S_{1}\right)
$$

We conclude this subsection by two properties of the principal symbol map linked to the composition and to the commutator of differential operators. For all $k, l \in \mathbb{N}$, we have:

$$
\begin{aligned}
& \sigma_{k+l}(A \circ B)=\sigma_{k}(A) \sigma_{l}(B), \\
& \sigma_{k+l-1}([A, B])=\left\{\sigma_{k}(A), \sigma_{l}(B)\right\},
\end{aligned}
$$

where $A$ and $B$ are elements of $\mathcal{D}(M)$ of order $k$ and $l$ respectively. 


\subsection{Pseudo-Riemannian and conformal geometry}

Let $(M, \mathrm{~g})$ be a pseudo-Riemannian manifold. The isometries $\Phi$ of $(M, \mathrm{~g})$ are the diffeomorphisms of $M$ that preserve the metric g, i.e. $\Phi^{*} \mathrm{~g}=\mathrm{g}$. Their infinitesimal counterparts $X \in \operatorname{Vect}(M)$ are called Killing vector fields, they satisfy $L_{X} \mathrm{~g}=0$, with $L_{X} \mathrm{~g}$ the Lie derivative of $g$ along $X$.

Given the Levi-Civita connection $\nabla$ corresponding to the metric g, the Riemannian curvature tensor, which reads as $\mathrm{R}_{a b}{ }^{c} d$ in abstract index notation, is given by $\left[\nabla_{a}, \nabla_{b}\right] v^{c}=\mathrm{R}_{a b}{ }^{c} v^{d}$ for a tangent vector field $v^{c}$. Then, one gets the Ricci tensor by taking a trace of the Riemann tensor, which is indicated by repeated indices: $\mathrm{Ric}_{b d}=\mathrm{R}_{a b}{ }_{d}{ }_{d}$. By contraction with the metric, the Ricci tensor leads to the scalar curvature $\mathrm{Sc}=\mathrm{g}^{a b} \mathrm{Ric}_{a b}$.

A conformal structure on a smooth manifold $M$ is given by the conformal class [g] of a pseudoRiemannian metric g, where two metrics g and $\hat{g}$ are conformally related if $\hat{g}=e^{2 \Upsilon} \mathrm{g}$, for some function $\Upsilon \in \mathcal{C}^{\infty}(M)$. The conformal diffeomorphisms $\Phi$ of $(M,[\mathrm{~g}])$ are those which preserve the conformal structure [g], i.e. there exists $\Upsilon \in \mathcal{C}^{\infty}(M)$ such that $\Phi^{*} \mathrm{~g}=e^{2 \Upsilon} \mathrm{g}$. Their infinitesimal counterparts $X \in \operatorname{Vect}(M)$ are called conformal Killing vector fields, they satisfy $L_{X} \mathrm{~g}=f_{X} \mathrm{~g}$, for some function $f_{X} \in \mathcal{C}^{\infty}(M)$.

Let $\left(x^{i}, p_{i}\right)$ be a canonical coordinate system on $T^{*} M$. If $M$ is endowed with a metric $\mathrm{g}$, we define the metric symbol and the trace operator by, respectively,

$$
H=\mathrm{g}^{i j} p_{i} p_{j} \quad \text { and } \quad \operatorname{Tr}=\mathrm{g}_{i j} \partial_{p_{i}} \partial_{p_{j}}
$$

Note that the symbol $\left|\operatorname{Vol}_{\mathrm{g}}\right|^{2 / n} H \in \mathcal{S}_{2 / n}$ and the operator $\left|\operatorname{Vol}_{\mathrm{g}}\right|^{-2 / n} \operatorname{Tr}: \mathcal{S}_{\delta} \rightarrow \mathcal{S}_{\delta-2 / n}$ are conformally invariant. In consequence, we get a conformally invariant decomposition

$$
S^{k} T M=\bigoplus_{0 \leq 2 s \leq k} S^{k, s} T M
$$

where $S \in \mathcal{S}^{k, s}(M):=\Gamma\left(S^{k, s} T M\right)$ is of the form $S=H^{s} S_{0}$ with $\operatorname{Tr} S_{0}=0$.

\subsection{The conformal Laplacian}

Starting from a pseudo-Riemannian manifold $(M, \mathrm{~g})$ of dimension $n$, one can define the Yamabe Laplacian, acting on functions, in the following way:

$$
\Delta_{Y}:=\nabla_{a} \mathrm{~g}^{a b} \nabla_{b}-\frac{n-2}{4(n-1)} \mathrm{Sc}
$$

where $\nabla$ denotes the Levi-Civita connection of $\mathrm{g}$ and Sc the scalar curvature. For the conformally related metric $\hat{\mathrm{g}}=e^{2 \Upsilon} \mathrm{g}$, the associated Yamabe Laplacian is given by

$$
\widehat{\Delta_{Y}}=e^{-\frac{n+2}{2} \Upsilon} \circ \Delta_{Y} \circ e^{\frac{n-2}{2} \Upsilon} \text {. }
$$

According to the transformation law $\left|\operatorname{Vol}_{\hat{\mathrm{g}}}\right|=e^{n \Upsilon}\left|\operatorname{Vol}_{\mathrm{g}}\right|$ and to the diagram (2.2), this translates into the conformal invariance of $\Delta_{Y}$ viewed as an element of $\mathcal{D}_{\lambda_{0}, \mu_{0}}(M)$, for the specific weights

$$
\lambda_{0}=\frac{n-2}{2 n}, \quad \mu_{0}=\frac{n+2}{2 n} \quad \text { and } \quad \delta_{0}=\mu_{0}-\lambda_{0}=\frac{2}{n} .
$$

Thus, the data of a conformal manifold $(M,[\mathrm{~g}])$ is enough to define $\Delta_{Y} \in \mathcal{D}_{\lambda_{0}, \mu_{0}}(M)$. We write it below as $\Delta_{Y}^{M}(\mathrm{~g})$ and we refer to it as the Yamabe or conformal Laplacian. One easily gets 
Proposition 2.1. The conformal Laplacian is a natural conformally invariant operator, i.e.

- it satisfies the naturality condition:

$$
\Delta_{Y}^{N}\left(\Phi^{*} \mathrm{~g}\right)=\Phi^{*}\left(\Delta_{Y}^{M}(\mathrm{~g})\right)
$$

for all diffeomorphisms $\Phi: N \rightarrow M$ and for all pseudo-Riemannian metric $\mathrm{g}$ on $M$,

- it is conformally invariant, $\Delta_{Y}^{M}\left(e^{2 \Upsilon} \mathrm{g}\right)=\Delta_{Y}^{M}(\mathrm{~g})$ for all $\Upsilon \in \mathcal{C}^{\infty}(M)$.

More generally, a natural operator over pseudo-Riemannian manifolds is an operator that acts between natural bundles, is defined over any pseudo-Riemannian manifold $(M, \mathrm{~g})$ and satisfies an analogue of the naturality condition (2.8). It is said to be conformally invariant if it depends only on the conformal class of g. For a general study of natural operators in the pseudo-Riemannian setting, see the book [20].

From Proposition 2.1, we deduce that the conformal Laplacian $\Delta_{Y}$ is invariant under the action of conformal diffeomorphisms, which reads infinitesimally as

$$
L_{X}^{\mu_{0}} \circ \Delta_{Y}=\Delta_{Y} \circ L_{X}^{\lambda_{0}}
$$

for all conformal Killing vector fields $X$. Here, as introduced in (2.1), $L^{\lambda_{0}}$ and $L^{\mu_{0}}$ denote the Lie derivatives of $\lambda_{0^{-}}$and $\mu_{0^{-}}$-densities. If the manifold $(M,[\mathrm{~g}])$ is locally conformally flat, then, up to multiplication by a scalar, $\Delta_{Y}$ is the unique second order operator acting on densities which is invariant under the action (2.9) of conformal Killing vector fields.

\subsection{Natural and conformally invariant quantization}

Recall first the definition of a quantization on a smooth manifold $M$.

Definition 2.2. Let $\lambda, \mu \in \mathbb{R}$ and $\delta=\mu-\lambda$. A quantization on $M$ is a linear bijection $\mathcal{Q}_{\lambda, \mu}^{M}$ from the space of symbols $\mathcal{S}_{\delta}(M)$ to the space of differential operators $\mathcal{D}_{\lambda, \mu}(M)$ such that

$$
\sigma_{k}\left(\mathcal{Q}_{\lambda, \mu}^{M}(S)\right)=S, \quad \forall S \in \mathcal{S}_{\delta}^{k}(M), \quad \forall k \in \mathbb{N} .
$$

On locally conformally flat manifolds $(M,[\mathrm{~g}])$, for generic weights $\lambda, \mu$, there exists a unique conformally equivariant quantization [12], i.e. a unique quantization which intertwines the actions of the conformal Killing vector fields on $\mathcal{S}_{\delta}(M)$ and on $\mathcal{D}_{\lambda, \mu}(M)$. In the following, we need an extension of the conformally equivariant quantization to arbitrary conformal manifolds. This is provided by the notion of natural and conformally invariant quantization. The definition and the conjecture of the existence of such a quantization were given for the first time in [22].

Definition 2.3. A natural and conformally invariant quantization is the data for every pseudoRiemannian manifold $(M, \mathrm{~g})$ of a quantization $\mathcal{Q}_{\lambda, \mu}^{M}(\mathrm{~g})$, which satisfies

- the naturality condition:

$$
\mathcal{Q}_{\lambda, \mu}^{N}\left(\Phi^{*} \mathrm{~g}\right)\left(\Phi^{*} S\right)=\Phi^{*}\left(\mathcal{Q}_{\lambda, \mu}^{M}(\mathrm{~g})(S)\right), \quad \forall S \in \mathcal{S}_{\delta}(M),
$$

for all diffeomorphisms $\Phi: N \rightarrow M$ and for all pseudo-Riemannian metric g on $M$.

- the conformal invariance: $\mathcal{Q}_{\lambda, \mu}^{M}\left(e^{2 \Upsilon} \mathrm{g}\right)=\mathcal{Q}_{\lambda, \mu}^{M}(\mathrm{~g})$ for all $\Upsilon \in \mathcal{C}^{\infty}(M)$.

In the following we refer to a quantization map by $\mathcal{Q}_{\lambda, \mu}$, the dependence in the chosen pseudoRiemannian manifold $(M, \mathrm{~g})$ being understood. Accordingly, we drop the reference to $M$ in the spaces of densities $\mathcal{F}_{\lambda}$, symbols $\mathcal{S}_{\delta}$ and differential operators $\mathcal{D}_{\lambda, \mu}$. 
The concept of natural and conformally invariant quantization is an extension to quantizations of the more usual one of natural conformally invariant operator, introduced in the previous section. Restricting to conformally flat manifolds $(M,[\mathrm{~g}])$ and to $\Phi \in \operatorname{Diff}(M)$ preserving $[\mathrm{g}]$, the naturality condition (2.10) reads as conformal equivariance of the quantization map $\mathcal{Q}_{\lambda, \mu}$. Thus, the problem of the natural and conformally invariant quantization on an arbitrary manifold generalizes the problem of the conformally equivariant quantization on conformally flat manifolds.

Remark that the bundles $S^{k} T M$ are natural bundles over $(M,[\mathrm{~g}])$. Hence, one can consider natural and conformally invariant quantization restricted to the subspaces of symbols $\mathcal{S}_{\delta}^{k}$ or $\mathcal{S}_{\delta}^{\leq k}=\bigoplus_{j \leq k} \mathcal{S}_{\delta}^{j}$. In a first step, the proofs of the existence of a natural and conformally invariant quantization at the second and the third orders were given respectively in [13] and [23], together with explicit formulas. We provide the one at order 2, which we will need later on.

Theorem 2.4 ([13]). Let $\delta \notin\left\{\frac{2}{n}, \frac{n+2}{2 n}, 1, \frac{n+1}{n}, \frac{n+2}{n}\right\}$. A natural and conformally invariant quantization $\mathcal{Q}_{\lambda, \mu}: \mathcal{S}_{\delta}^{\leq 2} \rightarrow \mathcal{D}_{\lambda, \mu}^{2}$ is provided, on a pseudo-Riemannian manifold $(M, \mathrm{~g})$ of dimension $n$, by the formulas

$$
\begin{aligned}
\mathcal{Q}_{\lambda, \mu}(f)= & f, \\
\mathcal{Q}_{\lambda, \mu}(X)= & X^{a} \nabla_{a}+\frac{\lambda}{1-\delta}\left(\nabla_{a} X^{a}\right), \\
\mathcal{Q}_{\lambda, \mu}(S)= & S^{a b} \nabla_{a} \nabla_{b}+\beta_{1}\left(\nabla_{a} S^{a b}\right) \nabla_{b}+\beta_{2} \mathrm{~g}^{a b}\left(\nabla_{a} \operatorname{Tr} S\right) \nabla_{b} \\
& +\beta_{3}\left(\nabla_{a} \nabla_{b} S^{a b}\right)+\beta_{4} \mathrm{~g}^{a b} \nabla_{a} \nabla_{b}(\operatorname{Tr} S),+\beta_{5} \operatorname{Ric}_{a b} S^{a b}+\beta_{6} \operatorname{Sc}(\operatorname{Tr} S),
\end{aligned}
$$

where $f, X, S$ are symbols of degrees $0,1,2$ respectively and $\operatorname{Tr} S=\mathrm{g}_{a b} S^{a b}$. Moreover the coefficients $\beta_{i}$ entering the last formula are given by

$$
\begin{aligned}
& \beta_{1}=\frac{2(n \lambda+1)}{2+n(1-\delta)}, \\
& \beta_{2}=\frac{n(\lambda+\mu-1)}{(2+n(1-\delta))(2-n \delta)}, \\
& \beta_{3}=\frac{n \lambda(n \lambda+1)}{(1+n(1-\delta))(2+n(1-\delta))} \\
& \beta_{4}=\frac{n \lambda\left(n^{2} \mu(2-\lambda-\mu)+2(n \lambda+1)^{2}-n(n+1)\right)}{(1+n(1-\delta))(2+n(1-\delta))(2+n(1-2 \delta))(2-n \delta)}, \\
& \beta_{5}=\frac{n^{2} \lambda(\mu-1)}{(n-2)(1+n(1-\delta))}, \\
& \beta_{6}=\frac{n^{2} \lambda(\mu-1)(n \delta-2)}{(n-1)(n-2)(1+n(1-\delta))(2+n(1-2 \delta))} .
\end{aligned}
$$

In a second step, the proof of the existence of such a quantization, at an arbitrary order and for generic values of $\lambda, \mu$, was given in $[9,25,30]$ in different ways. We provide a slightly refined statement in the next section.

\subsection{Adjoint operation and quantization}

For all weights $\lambda \in \mathbb{R}$, there exists a non-degenerate symmetric bilinear pairing

$$
\begin{aligned}
& \mathcal{F}_{\lambda}^{c} \times \mathcal{F}_{1-\lambda}^{c} \rightarrow \mathbb{R}, \\
&(\varphi, \psi) \quad \mapsto \int_{M} \varphi \psi,
\end{aligned}
$$


where $\mathcal{F}_{\lambda}^{c}$ is the space of compactly supported $\lambda$-densities. On a manifold $M$, this pairing is $\operatorname{Diff}(M)$-invariant since 1-density is the right object for integration. In consequence, we can define an adjoint operation $*: \mathcal{D}_{\lambda, \mu} \rightarrow \mathcal{D}_{1-\mu, 1-\lambda}$ by

$$
\left(\varphi, D^{*} \psi\right)=(D \varphi, \psi)
$$

for all $\varphi \in \mathcal{F}_{\lambda}^{c}$ and $\psi \in \mathcal{F}_{1-\mu}^{c}$. We introduce the following subset of $\mathbb{R}^{2}$,

$$
I=\left\{(\lambda, \mu) \in \mathbb{R}^{2} \mid \mu-\lambda \notin \frac{1}{2 n}(\mathbb{N} \backslash\{0\})\right\} \cup\left\{\left(\lambda_{0}, \mu_{0}\right)\right\},
$$

where $\lambda_{0}=\frac{n-2}{2 n}$ and $\mu_{0}=\frac{n+2}{2 n}$ are the weights of the conformal Laplacian (see (2.7)). The set $I$ is stable under the involutive map $(\lambda, \mu) \mapsto(1-\mu, 1-\lambda)$. Note that

$$
\sigma_{2}\left(\Delta_{Y}\right)=\left|\operatorname{Vol}_{\mathrm{g}}\right|^{\delta_{0}} H
$$

where $\delta_{0}=\mu_{0}-\lambda_{0}$ and $H=\mathrm{g}^{i j} p_{i} p_{j}$ in canonical coordinates. The proof of existence of a natural and conformally invariant quantization $\mathcal{Q}_{\lambda, \mu}$ in [30] leads easily to the following statement.

Theorem 2.5. There exists a family $\left(\mathcal{Q}_{\lambda, \mu}\right)_{(\lambda, \mu) \in I}$ of natural and conformally invariant quantizations that satisfies:

- the reality condition:

$$
\mathcal{Q}_{\lambda, \mu}(S)^{*}=(-1)^{k} \mathcal{Q}_{1-\mu, 1-\lambda}(S), \quad \forall S \in \mathcal{S}_{\delta}^{k}, \quad \forall(\lambda, \mu) \in I,
$$

- the factorization property:

$$
\begin{array}{ll}
\mathcal{Q}_{\lambda_{0}, \lambda_{0}}\left(\left|\operatorname{Vol}_{\mathrm{g}}\right|{ }^{\delta_{0}} H S\right)=\mathcal{Q}_{\mu_{0}, \lambda_{0}}(S) \circ \Delta_{Y}, & \forall S \in \mathcal{S}_{-\delta_{0}}^{k}, \\
\mathcal{Q}_{\mu_{0}, \mu_{0}}\left(\left|\operatorname{Vol}_{\mathrm{g}}\right|^{\delta_{0}} H S\right)=\Delta_{Y} \circ \mathcal{Q}_{\mu_{0}, \lambda_{0}}(S), & \forall S \in \mathcal{S}_{-\delta_{0}}^{k},
\end{array}
$$

- the restriction of $\mathcal{Q}_{\lambda, \mu}$ to $\mathcal{S}_{\bar{\delta}}^{\leq 2}$ is given by the formulas in (2.11) if $(\lambda, \mu) \in I \backslash\left\{\left(\lambda_{0}, \mu_{0}\right)\right\}$.

Proof. We prove the theorem in four steps.

In [30, Theorem 4.4], one of us determines that for $(\lambda, \mu) \in I$ there exists a natural and conformally invariant quantization map $\mathcal{Q}_{\lambda, \mu}^{\prime \prime \prime}$.

From the above family of quantizations $\left(\mathcal{Q}_{\lambda, \mu}^{\prime \prime \prime}\right)_{(\lambda, \mu) \in I}$, we define $\left(\mathcal{Q}_{\lambda, \mu}^{\prime \prime}\right)_{(\lambda, \mu) \in I}$ by

$$
\mathcal{Q}_{\lambda, \mu}^{\prime \prime}: S \mapsto \frac{1}{2}\left(\mathcal{Q}_{\lambda, \mu}^{\prime \prime \prime}(S)+(-1)^{k} \mathcal{Q}_{1-\mu, 1-\lambda}^{\prime \prime \prime}(S)^{*}\right), \quad \forall S \in \mathcal{S}_{\delta}^{k}, \quad \forall(\lambda, \mu) \in I .
$$

The maps $\mathcal{Q}_{\lambda, \mu}^{\prime \prime}$ are again natural and conformally invariant quantizations. Indeed, the adjoint operation $*$ is natural, does not depend of the choice of metric on $M$ and satisfies

$$
\sigma_{k}\left(D^{*}\right)=(-1)^{k} \sigma_{k}(D)
$$

for all differential operators $D$ of order $k$. The newly defined quantization maps clearly satisfy the property (2.13) since $*$ is an involution.

For $(\lambda, \mu) \in I \backslash\left\{\left(\lambda_{0}, \lambda_{0}\right),\left(\mu_{0}, \mu_{0}\right)\right\}$, we define $\mathcal{Q}_{\lambda, \mu}^{\prime}:=\mathcal{Q}_{\lambda, \mu}^{\prime \prime}$. On the space of traceless symbols we set $\mathcal{Q}_{\lambda_{0}, \lambda_{0}}^{\prime}:=\mathcal{Q}_{\lambda_{0}, \lambda_{0}}^{\prime \prime}$ and $\mathcal{Q}_{\mu_{0}, \mu_{0}}^{\prime}:=\mathcal{Q}_{\mu_{0}, \mu_{0}}^{\prime \prime}$. We extend both maps to the whole symbol space by the formulas in (2.14). They are clearly still natural and conformally invariant and satisfy the reality condition $(2.13)$.

For all $(\lambda, \mu) \in I \backslash\left\{\left(\lambda_{0}, \mu_{0}\right)\right\}$, we denote by $\mathcal{Q}_{\lambda, \mu}$ the natural and conformally invariant quantizations restricted to $\mathcal{S}_{\delta}^{\leq 2}$, given by the formulas (2.11). A direct computation shows that they satisfy the reality condition (2.13) and the factorization property $(2.14)$. For $(\lambda, \mu)=\left(\lambda_{0}, \mu_{0}\right)$, we set $\mathcal{Q}_{\lambda_{0}, \mu_{0}}:=\mathcal{Q}_{\lambda_{0}, \mu_{0}}^{\prime}$ on $\mathcal{S}_{\delta_{0}}^{\leq 2}$. We extend then the quantizations $\mathcal{Q}_{\lambda, \mu}$ (where $(\lambda, \mu) \in I$ ) to the whole symbol space by setting $\mathcal{Q}_{\lambda, \mu}:=\mathcal{Q}_{\lambda, \mu}^{\prime}$ on $\mathcal{S}_{\bar{\delta}}^{\geq 3}$, for all $(\lambda, \mu) \in I$. 
In the following, the quantization maps $\mathcal{Q}_{\lambda, \mu}$ that we will use are always taken from a family $\left(\mathcal{Q}_{\lambda, \mu}\right)_{(\lambda, \mu) \in I}$ provided by Theorem 2.5. In fact, we will need only four of them, namely: $\mathcal{Q}_{\lambda_{0}, \lambda_{0}}$, $\mathcal{Q}_{\mu_{0}, \mu_{0}}, \mathcal{Q}_{\lambda_{0}, \mu_{0}}, \mathcal{Q}_{\mu_{0}, \lambda_{0}}$. With such a convention, it is worth noticing that the conformal Laplacian can be obtained as

$$
\Delta_{Y}=\mathcal{Q}_{\lambda_{0}, \mu_{0}}\left(\left|\operatorname{Vol}_{\mathrm{g}}\right|^{\delta_{0}} H\right)
$$

The conformal invariance of the symbol $\left|\mathrm{Vol}_{\mathrm{g}}\right|^{\delta_{0}} \mathrm{H}$ translates into the conformal invariance of $\Delta_{Y}$.

\section{On particular conformally invariant operators}

First, we introduce notation for classical objects of the pseudo-Riemannian and conformal geometries and recall basic facts about natural and conformally invariant operators. Then, we classify the natural conformally invariant operators between particular subspaces of symbols.

\subsection{More on pseudo-Riemannian and conformal geometry}

We complete here Section 2.2, and use freely the notation introduced there.

First, we work over a pseudo-Riemannian manifold. The Riemann tensor admits the following decomposition

$$
\mathrm{R}_{a b d}{ }^{c}=\mathrm{C}_{a b d}{ }^{c}+2 \delta_{[a}^{c} \mathrm{P}_{b] d}+2 \mathrm{~g}_{d[b} \mathrm{P}_{a]}{ }^{c},
$$

where $\mathrm{C}_{a b}{ }^{c} d$ is the totally trace-free Weyl curvature, $\mathrm{P}_{a b}=\frac{1}{n-2}\left(\mathrm{Ric}_{a b}-\frac{1}{2(n-1)} \mathrm{Sc} \mathrm{g}_{a b}\right)$ is the Schouten tensor, $\delta_{a}^{b}$ is the Kronecker delta and square brackets denote antisymmetrization of enclosed indices. The Weyl tensor $\mathrm{C}_{a b c d}$ is zero for the dimension $n=3$. Note also that $\mathrm{C}_{a b c d}$ obeys the same symmetries of indices as $\mathrm{R}_{a b c d}$ does. Further curvature quantities we shall need are

$$
\mathrm{J}=\mathrm{g}^{a b} \mathrm{P}_{a b} \quad \text { and } \quad \mathrm{A}_{a b c}=2 \nabla_{[b} \mathrm{P}_{c] a}
$$

where $\mathrm{A}_{a b c}$ is the Cotton-York tensor and $\mathrm{J}$ is related to the scalar curvature via $\mathrm{J}=\frac{1}{2(n-1)}$ Sc. Bianchi identities have the from $\mathrm{R}_{[a b c] d}=0$ and $\nabla_{[a} \mathrm{R}_{b c] d e}=0$ and lead to

$$
(n-3) \mathrm{A}_{a b c}=\nabla_{r} \mathrm{C}_{b c}{ }^{r} a \quad \text { and } \quad \nabla_{b} \mathrm{P}_{a}^{b}=\nabla_{a} \mathrm{~J} .
$$

Second, we consider a conformal manifold $(M,[\mathrm{~g}])$. The Weyl tensor $\mathrm{C}_{a b}{ }^{c} d$ is a conformal invariant, i.e. it does not depend on the choice of the representative metric from $[\mathrm{g}]$. The same is true for $\mathrm{A}_{a b c}$ in the dimension 3. Further, a choice of metric provides a canonical trivialization of the bundle of $\lambda$-densities $F_{\lambda}$ via the global section $\left|\operatorname{Vol}_{\mathrm{g}}\right|^{\lambda}$ (see Section 2.1). According to

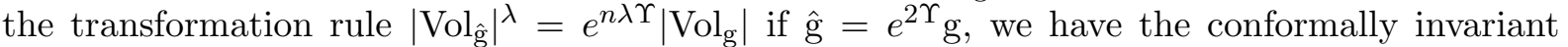
object $\mathbf{g}_{a b}=\mathrm{g}_{a b} \otimes\left|\mathrm{Vol}_{\mathrm{g}}\right|^{-\frac{2}{n}}$, termed conformal metric, with the inverse $\mathbf{g}^{a b}$ in $\Gamma\left(S^{2} T M\right) \otimes \mathcal{F}_{2 / n}$, see e.g. [1] for details. (Note that the space of densities $\mathcal{E}[w]$ in [1] corresponds to $\mathcal{F}_{-w / n}$ in our notation.) The conformal metric gives a conformally invariant identification $T M \cong$ $T^{*} M \otimes F_{-2 / n}$. In other words, we can raise and lower indices, with expense of the additional density, in a conformally invariant way. For example, we get $\mathrm{C}_{a b c d} \in \Gamma\left(S^{2}\left(\Lambda^{2} T^{*} M\right)\right) \otimes \mathcal{F}_{-2 / n}$. Note also that $\mathbf{g}_{a b}$ and $\mathbf{g}^{a b}$ are parallel for any choice of a Levi-Civita connection from the conformal class. 


\subsection{Description of natural conformally invariant operators}

Now we recall basic facts about natural and conformally invariant operators. Every natural operator on the Riemannian structure $(M, \mathrm{~g})$ between natural bundles $V_{1}$ and $V_{2}$ is a linear combination of terms of the form

$$
\underbrace{\mathrm{g}^{-1} \cdots \mathrm{g}^{-1}}_{r_{1}} \underbrace{\mathrm{g} \cdots \mathrm{g}}_{r_{2}} \underbrace{\left(\nabla^{\left(i_{1}\right)} \mathrm{R}\right) \cdots\left(\nabla^{\left(i_{s}\right)} \mathrm{R}\right)}_{s} \underbrace{\nabla \cdots \nabla}_{t} f
$$

to which one applies a $\mathrm{GL}(n)$-invariant operation

$$
\Gamma\left(\bigotimes^{2 r_{1}+s} T M \otimes \bigotimes^{2 r_{2}+3 s+i+t} T^{*} M \otimes V_{1}\right) \longrightarrow \Gamma\left(V_{2}\right)
$$

Here $f \in \Gamma\left(V_{1}\right), \mathrm{g}^{-1}$ stands for the inverse of the metric $\mathrm{g}, \nabla^{\left(i_{j}\right)}$ denotes the $i_{j}$ th iterated covariant derivative where $i_{j} \geq 0$, abstract indices are omitted, $i=i_{1}+\cdots+i_{s}$, and $\nabla$ and $\mathrm{R}$ correspond to the choice of the metric $g$. The existence of a $\operatorname{GL}(n)$-invariant operation (3.3) gives in general constraints on the possible values of $r_{1}, r_{2}, s, t, i$. See [20] for details.

A natural operator on $(M, \mathrm{~g})$ is conformally invariant if it does not depend on the choice of metric in the conformal class. Then, it defines a natural operator on the conformal structure $(M,[\mathrm{~g}])$. It is convenient to use the conformal metric $\mathbf{g}$ instead of $\mathrm{g}$ and the inverse $\mathbf{g}^{-1}$ instead of $\mathrm{g}^{-1}$ in (3.2) since they are conformally invariant, namely

$$
\begin{aligned}
& \underbrace{\mathbf{g}^{-1} \cdots \mathbf{g}^{-1}}_{r_{1}} \underbrace{\mathbf{g} \cdots \mathbf{g}}_{r_{2}} \underbrace{\left(\nabla^{\left(i_{1}\right)} \mathrm{R}\right) \cdots\left(\nabla^{\left(i_{s}\right)} \mathrm{R}\right)}_{s} \underbrace{\nabla \cdots \nabla}_{t} f \\
& \quad \in \Gamma\left(\bigotimes^{2 r_{1}+s} T M \otimes \bigotimes^{2 r_{2}+3 s+i+t} T^{*} M \otimes V_{1} \otimes F_{\frac{2}{n}\left(r_{1}-r_{2}\right)}\right)
\end{aligned}
$$

for $f \in \Gamma\left(V_{1}\right)$. It is generally a difficult problem to determine which linear combinations of terms as in (3.2), together with suitable projections as in (3.3), give rise to a conformally invariant operator. We shall need details only in specific cases.

\subsection{Conformally invariant operators on the symbol space}

This section concerns existence and uniqueness of natural and conformally invariant operators of certain type. The first one is well-known and can be obtained as an easy consequence of [17], or deduced from the general work [10] on curved BGG-sequences. We present a detailed proof to demonstrate the technique which is used (in much more complicated setting) later in the proof of Proposition 3.2.

Recall first that $\Gamma\left(S^{k, 0} T M\right)$ is the space of trace-less symmetric $k$-tensors. In terms of the abstract index notation, a section $f$ of $\otimes^{k} T M$ is denoted by $f^{a_{1} \ldots a_{k}}$. In the following, we write $f^{\left[a_{1} \ldots a_{k}\right]}, f^{\left(a_{1} \ldots a_{k}\right)}$ and $f^{\left(a_{1} \ldots a_{k}\right)_{0}}$ for the projections of $f$ to $\Gamma\left(\Lambda^{k} T M\right), \Gamma\left(S^{k} T M\right)$ and $\Gamma\left(S^{k, 0} T M\right)$, respectively. Similar notation will be used for covariant indices.

Proposition 3.1. Up to multiplication by a scalar, there exists a unique natural conformally invariant operator $\mathcal{S}_{0}^{k} \rightarrow \mathcal{S}_{2 / n}^{k+1}$. It is given by the conformal Killing operator $\mathbf{G}$, such that for all $f \in \mathcal{S}_{0}^{k}$,

$$
(\mathbf{G}(f))^{a_{0} \ldots a_{k}}=\nabla^{\left(a_{0}\right.} f^{\left.a_{1} \ldots a_{k}\right)_{0}} .
$$

Proof. Identifying $\mathcal{S}_{0}^{k}$ and $\mathcal{S}_{2 / n}^{k+1}$ with corresponding spaces of sections of symmetric tensors, we consider natural and conformally invariant operators $\Gamma\left(S^{k} T M\right) \rightarrow \Gamma\left(S^{k+1} T M \otimes F_{2 / n}\right)$. By 
naturality, such operators are linear combinations of terms in (3.2) with $V_{1}=S^{k} T M$, composed with $\mathrm{GL}_{n}$-invariant maps

$$
\bigotimes^{2 r_{1}+s} T M \otimes \bigotimes^{2 r_{2}+3 s+i+t} T^{*} M \otimes S^{k} T M \otimes F_{\frac{2}{n}\left(r_{1}-r_{2}\right)} \longrightarrow S^{k+1} T M \otimes F_{2 / n}
$$

Explicitly, those maps may consist of: contracting covariant and contravariant indices, projecting the covariant and contravariant tensors on tensors of prescribed symmetry type (given by a Young diagram) and tensorizing with the density $\left|\mathrm{Vol}_{\mathrm{g}}\right|^{\delta}$ for arbitrary $\delta \in \mathbb{R}$. The conformal invariance does not allow for the last operation, hence $r_{1}-r_{2}=1$. The difference between the number of covariant and contravariant indices is a constant therefore $\left(2 r_{1}+s+k\right)-\left(2 r_{2}+3 s+i+t\right)=$ $k+1$, i.e. $2 s+i+t=1$. This means $s=i=0$ and $t=1$. The sought operators are then first order (gradient) natural operators and using moreover the conformal invariance, the statement follows from the classification in [17].

The next proposition is a crucial technical tool in the following.

Proposition 3.2. Every natural conformally invariant operator $\mathcal{S}_{0}^{k, 0} \rightarrow \mathcal{S}_{2 / n}^{k-1}$ has its target space in $\mathcal{S}_{2 / n}^{k-1,0} \subseteq \mathcal{S}_{2 / n}^{k-1}$. The space of natural conformally invariant operators $\mathcal{S}_{0}^{k, 0} \rightarrow \mathcal{S}_{2 / n}^{k-1,0}$ on a pseudo-Riemannian manifold $(M, \mathrm{~g})$ is at most two-dimensional and depends on $k \in \mathbb{N}$ as follows.

(i) This space is trivial for $k=1$.

(ii) If $k=2$ or $n=3$, this space is one-dimensional and generated by the operator $\mathbf{F}$ such that, for all $f \in \mathcal{S}_{0}^{k, 0}$,

$$
(\mathbf{F}(f))^{a_{1} \ldots a_{k-1}}=\mathrm{C}_{s t}^{r}{ }^{\left(a_{1}\right.} \nabla_{r} f^{\left.a_{2} \ldots a_{k-1}\right)_{0} s t}-(k+1) \mathrm{A}_{s t}{ }^{\left(a_{1}\right.} f^{\left.a_{2} \ldots a_{k-1}\right)_{0} s t} .
$$

(iii) If $k=3$ and $n>3$, this space is two-dimensional and generated by two operators, $\mathbf{F}_{1}$ and $\mathbf{F}_{2}$, such that for all $f \in \mathcal{S}_{0}^{k, 0}$,

$$
\begin{aligned}
& \left(\mathbf{F}_{1}(f)\right)^{a_{1} \ldots a_{k-1}}=(\mathbf{F}(f))^{a_{1} \ldots a_{k-1}}+\frac{k-2}{n+2 k-2} \mathrm{C}^{\left(a_{1}{ }_{r}{ }^{a_{2}}{ }_{s} \nabla_{t}\right.} f^{\left.a_{3} \ldots a_{k-1}\right)_{0} r s t}, \\
& \left(\mathbf{F}_{2}(f)\right)^{a_{1} \ldots a_{k-1}}=4 \mathrm{C}^{\left(a_{1}{ }_{r} a_{s}\right.} \nabla_{t} f^{\left.a_{3} \ldots a_{k-1}\right)_{0} r s t}+(n+2 k-2)\left(\nabla_{r} \mathrm{C}_{s}{ }^{\left(a_{1}\right.}{ }_{t} a_{2}\right) f^{\left.a_{3} \ldots a_{k-1}\right)_{0} r s t} \\
& +2(n+2 k-2) \mathrm{A}_{r s}{ }^{\left(a_{1}\right.} f^{\left.a_{2} \ldots a_{k-1}\right)_{0} r s} \text {. }
\end{aligned}
$$

Remark 3.3. Let $\left(x^{i}, p_{i}\right)$ be a canonical coordinate system on $T^{*} M$. We can then write the operators $\mathbf{G}$ and $\mathbf{F}$ as follows on $\mathcal{S}_{0}^{k}$

$$
\mathbf{G}=\Pi_{0} \circ\left(\mathbf{g}^{i j} p_{i} \nabla_{j}\right) \quad \text { and } \quad \mathbf{F}=\Pi_{0} \circ \mathbf{g}^{i m} p_{i} \partial_{p_{j}} \partial_{p_{l}}\left(\mathrm{C}^{k}{ }_{j l m} \nabla_{k}-(k+1) \mathrm{A}_{j l m}\right),
$$

where $\Pi_{0}: \mathcal{S}_{0}^{k-1} \rightarrow \mathcal{S}_{2 / n}^{k-1,0}$ is the canonical projection on trace-less symbols. Actually, we will see in the sequel that the conformal Killing operator $\mathbf{G}$ can be used to define the conformal Killing tensors whereas the operator $\mathbf{F}$ occurs in the computation of the obstruction to the existence of conformal symmetries of $\Delta_{Y}$.

Let us note that the proof of Proposition 3.2 is long, technical and interesting rather for experts in conformal geometry. The reader interested mainly in results about symmetries can continue the reading in Section 4 (details from the proof will not be needed there). 
Proof. We study natural and conformally invariant operators $\Gamma\left(S^{k, 0} T M\right) \rightarrow \Gamma\left(S^{k-1} T M \otimes F_{2 / n}\right)$. In the first part of the proof we consider the naturality and in the second part the conformal invariance.

I. Naturality. We start in a similar way as in the proof of Proposition 3.1. By naturality, the considered operators are linear combinations of terms in (3.2) composed with $\mathrm{GL}_{n}$-invariant maps

$$
\bigotimes^{2 r_{1}+s} T M \otimes \bigotimes^{2 r_{2}+3 s+i+t} T^{*} M \otimes S^{k} T M \otimes F_{\frac{2}{n}\left(r_{1}-r_{2}\right)} \longrightarrow S^{k-1} T M \otimes F_{2 / n} .
$$

The conformal invariance of the discussed operators leads to $r_{1}-r_{2}=1$ and the $\mathrm{GL}_{n}$-invariance of the maps above imposes $\left(2 r_{1}+s+k\right)-\left(2 r_{2}+3 s+i+t\right)=k-1$, i.e. $2 s+i+t=3$. This means either $s=i=0, t=3$ or $s=i=1, t=0$ or $s=t=1, i=0$. Hence, omitting abstract indices, the natural operators $\Gamma\left(S^{k, 0} T M\right) \rightarrow \Gamma\left(S^{k-1} T M \otimes F_{2 / n}\right)$ are a linear combination of terms

$$
\underbrace{\mathbf{g}^{-1} \cdots \mathbf{g}^{-1}}_{r+1} \underbrace{\mathbf{g} \cdots \mathbf{g}}_{r} \nabla \nabla \nabla f, \quad \underbrace{\mathbf{g}^{-1} \cdots \mathbf{g}^{-1}}_{r+1} \underbrace{\mathbf{g} \cdots \mathbf{g}}_{r} \mathrm{R} \nabla f, \quad \underbrace{\mathbf{g}^{-1} \cdots \mathbf{g}^{-1}}_{r+1} \underbrace{\mathbf{g} \cdots \mathbf{g}}_{r}(\nabla \mathrm{R}) f
$$

where $r \geq 0$ and $f \in \Gamma\left(S^{k, 0} T M\right)$, each of which is followed by a GL(n)-invariant projection to $\Gamma\left(S^{k-1} T M \otimes F_{2 / n}\right)$. Irreducible components of the target bundle $S^{k-1} T M \otimes F_{2 / n}$ are

$$
S^{k-1,0} T M \otimes F_{2 / n}, \quad S^{k-3,0} T M, \quad S^{k-5,0} T M \otimes F_{-2 / n}, \quad \ldots,
$$

but since $f$ is trace-free, one easily verifies from (3.7) that only possible target bundles are $S^{k-1,0} T M \otimes F_{2 / n}$ and $S^{k-3,0} T M$. In other words, in the expressions (3.7), one can restrict to $r=0$.

It remains to describe possible $\mathrm{GL}(n)$-invariant projections of the terms in (3.7) in details. Using the decomposition (3.1) of $\mathrm{R}$ into Weyl and Schouten tensors, they split into five terms: $\mathbf{g}^{-1} \nabla \nabla \nabla f, \mathbf{g}^{-1} \mathrm{C} \nabla f, \mathbf{g}^{-1}(\nabla \mathrm{C}) f, \mathbf{g}^{-1} \mathrm{P} \nabla f$ and $\mathbf{g}^{-1}(\nabla \mathrm{P}) f$.

We shall start with natural operators $\Gamma\left(S^{k, 0} T M\right) \rightarrow \Gamma\left(S^{k-1,0} T M \otimes F_{2 / n}\right)$. In this situation, at least one of the two indices above $\mathbf{g}^{-1}$ in the expression of the operator has to be contracted with a covariant index. For an operator of type $\mathbf{g}^{-1} \nabla \nabla \nabla f$, the two resulting operators are (up to the order of covariant derivatives) respectively

$$
\nabla^{r} \nabla_{r} \nabla_{s} f^{a_{1} \ldots a_{k-1} s}, \quad \nabla^{\left(a_{1}\right.} \nabla_{s} \nabla_{t} f^{\left.a_{2} \ldots a_{k-1}\right)_{0} s t} .
$$

Since the change of the order of covariant derivatives gives rise to curvature operators of the form $\mathbf{g}^{-1} \mathrm{R} \nabla f$ and $\mathbf{g}^{-1}(\nabla \mathrm{R}) f$, the previous display is sufficient for operators of type $\mathbf{g}^{-1} \nabla \nabla \nabla f$. Using that $\mathrm{C}$ is completely trace-free and $(n-3) \mathrm{A}_{a b c}=\nabla_{r} \mathrm{C}_{b c}{ }^{r} a$, the different possibilities of contraction of indices for the expressions $\mathbf{g}^{-1} \mathrm{C} \nabla f$ and $\mathbf{g}^{-1}(\nabla \mathrm{C}) f$ lead to the operators

$$
\begin{aligned}
& \mathrm{C}^{r}{ }_{s t}^{\left(a_{1}\right.} \nabla_{r} f^{\left.a_{2} \ldots a_{k-1}\right)_{0} s t}, \quad \mathrm{C}^{\left(a_{1}\right.}{ }_{r}{ }_{2}{ }_{s} \nabla_{t} f^{\left.a_{3} \ldots a_{k-1}\right)_{0} r s t}, \\
& \left(\nabla_{r} \mathrm{C}_{s}{ }^{\left(a_{1}\right.} t^{a_{2}}\right) f^{\left.a_{3} \ldots a_{k-1}\right)_{0} r s t}, \quad \mathrm{~A}_{r s}{ }^{\left(a_{1}\right.} f^{\left.a_{2} \ldots a_{k-1}\right)_{0} r s} .
\end{aligned}
$$

Thanks to the decomposition of $\mathrm{P}$ into irreducible components and to the equality $\nabla_{a} \mathrm{P}_{b}^{a}=\nabla_{b} J$, we see that the different configurations of indices in the expressions $\mathrm{g}^{-1} \mathrm{P} \nabla f$ and $\mathrm{g}^{-1}(\nabla \mathrm{P}) f$ give rise to the operators

$$
\begin{aligned}
& \mathrm{P}_{(r s)_{0}} \nabla^{r} f^{s a_{1} \ldots a_{k-1}}, \quad \mathrm{P}_{(r t)_{0}} \mathbf{g}^{t\left(a_{1}\right.} \nabla_{s} f^{\left.a_{2} \ldots a_{k-1}\right)_{0} r s}, \quad \mathrm{P}_{r s} \nabla^{\left(a_{1}\right.} f^{\left.a_{2} \ldots a_{k-1}\right)_{0} r s}, \\
& \mathrm{~J} \nabla_{r} f^{a_{1} \ldots a_{k-1} r}, \quad\left(\nabla_{(r} \mathrm{P}_{s t)_{0}}\right) \mathbf{g}^{t\left(a_{1}\right.} f^{\left.a_{2} \ldots a_{k-1}\right)_{0} r s}, \quad\left(\nabla_{r} \mathrm{~J}\right) f^{a_{1} \ldots a_{k-1} r} .
\end{aligned}
$$

Hence all natural operators $\Gamma\left(S^{k, 0} T M\right) \rightarrow \Gamma\left(S^{k-1,0} T M \otimes F_{2 / n}\right)$ are linear combinations of terms in $(3.8)-(3.10)$. 
A similar discussion can be applied to natural operators $\Gamma\left(S^{k, 0} T M\right) \rightarrow \Gamma\left(S^{k-3,0} T M\right)$. In this situation, none of the two indices of $\mathbf{g}^{-1}$ is contracted in the expressions (3.7). Reasoning as above, using the properties of symmetry of $\mathrm{C}$ and the fact that $\mathrm{C}$ and $f$ are trace-free, we obtain (since the target bundle is now $S^{k-3,0} T M$ ) a simpler list of possible terms:

$$
\nabla_{r} \nabla_{s} \nabla_{t} f^{a_{1} \ldots a_{k-3} r s t}, \quad \mathrm{P}_{(r s)_{0}} \nabla_{t} f^{a_{1} \ldots a_{k-3} r s t}, \quad\left(\nabla_{r} \mathrm{P}_{s t}\right) f^{a_{1} \ldots a_{k-3} r s t},
$$

for $k \geq 3$. Hence all natural operators $\Gamma\left(S^{k, 0} T M\right) \rightarrow \Gamma\left(S^{k-3,0} T M\right)$ are linear combinations of terms in (3.11).

II. Conformal invariance. We shall denote quantities corresponding to the conformally related metric $\hat{\mathrm{g}}=e^{2 \Upsilon_{\mathrm{g}}}$ and the corresponding Levi-Civita connection $\hat{\nabla}$ by $\hat{\mathrm{R}}_{a b c d}, \hat{\mathrm{P}}_{a b}, \hat{\mathrm{J}}$ and $\hat{\mathrm{A}}_{a b c}$. (The Weyl tensor is missing here since $\hat{\mathrm{C}}_{a b c d}=\mathrm{C}_{a b c d}$.) This transformation is controlled by the one-form $\Upsilon_{a}=\nabla_{a} \Upsilon$, see e.g. [1] for details. Explicitly, one can compute that

$$
\begin{aligned}
& \widehat{\mathrm{P}}_{a b}=\mathrm{P}_{a b}-\nabla_{a} \Upsilon_{b}+\Upsilon_{a} \Upsilon_{b}-\frac{1}{2} \Upsilon^{r} \Upsilon_{r} \mathrm{~g}_{a b}, \\
& \widehat{\mathrm{J}}=\mathrm{J}-\nabla^{r} \Upsilon_{r}-\frac{n-2}{2} \Upsilon^{r} \Upsilon_{r} \quad \text { and } \quad \widehat{\mathrm{A}}_{a b c}=\mathrm{A}_{a b c}+\Upsilon_{r} \mathrm{C}_{b c}{ }^{r}{ }_{a}
\end{aligned}
$$

and also that

$$
\begin{aligned}
& \widehat{\nabla}_{(a} \widehat{\mathrm{P}}_{b c)_{0}}=\nabla_{(a} \mathrm{P}_{b c)_{0}}-\nabla_{(a} \nabla_{b} \Upsilon_{c)_{0}}+4 \Upsilon_{(a} \nabla_{b} \Upsilon_{c)_{0}}-4 \Upsilon_{(a} \Upsilon_{b} \Upsilon_{c)_{0}}-2 \Upsilon_{(a} \mathrm{P}_{b c)_{0}}, \\
& \widehat{\nabla}_{a} \widehat{\mathrm{J}}=\nabla_{a} \mathrm{~J}-\nabla_{a} \nabla^{r} \Upsilon_{r}-(n-2) \Upsilon^{r} \nabla_{r} \Upsilon_{a}+2 \Upsilon_{a} \nabla^{r} \Upsilon_{r}-2 \Upsilon_{a} \mathrm{~J}+(n-2) \Upsilon_{a} \Upsilon^{r} \Upsilon_{r}, \\
& \widehat{\nabla}_{\left({ }_{a} \mathrm{C}_{b}{ }^{d}{ }_{c)_{0}}{ }^{e}=\nabla_{(a} \mathrm{C}_{b}{ }^{d}{ }_{c)_{0}}{ }^{e}-4 \Upsilon_{(a} \mathrm{C}_{b}{ }^{d}{ }_{c)_{0}}{ }^{e}+2 \Upsilon_{r} \delta_{(a}\left({ }^{d} \mathrm{C}_{b}{ }^{e)}{ }_{c)}{ }^{r} .\right.\right.} .
\end{aligned}
$$

We shall start with operators $\Gamma\left(S^{k, 0} T M\right) \rightarrow \Gamma\left(S^{k-1,0} T M \otimes F_{2 / n}\right)$. First observe that the space of such natural and conformally invariant operators is trivial in the flat case $[4,5]$ hence the two terms of (3.8) cannot appear. We need to know how remaining terms in (3.9) and (3.10) transform under the conformal rescaling $\hat{g}=e^{2 \Upsilon} \mathrm{g}$. First observe that the rescaling of first order expressions we need is

$$
\begin{aligned}
& \widehat{\nabla}_{r} f^{a_{1} \ldots a_{k-1} r}=\nabla_{r} f^{a_{1} \ldots a_{k-1} r}+(n+2 k-2) \Upsilon_{r} f^{a_{1} \ldots a_{k-1} r}, \\
& \left.\widehat{\nabla}_{\left[b f_{c]}\right.}^{a_{1} \ldots a_{k-1}}=\nabla_{[b} f_{c]}^{a_{1} \ldots a_{k-1}}+(k+1) \Upsilon_{[b} f_{c]}^{a_{1} \ldots a_{k-1}}+(k-1) \Upsilon_{r} \delta_{[b}^{\left(a_{1}\right.} f_{c]} a_{2} \ldots a_{k-1}\right) r, \\
& \widehat{\nabla}^{\left(a_{1}\right.} f^{\left.a_{2} \ldots a_{k-1}\right)_{0}}{ }_{b c}=\nabla^{\left(a_{1}\right.} f^{\left.a_{2} \ldots a_{k-1}\right)_{0}} b c+2 \Upsilon^{\left(a_{1}\right.} f^{\left.a_{2} \ldots a_{k-1}\right)_{0}} b c-2 \Upsilon_{(b} f_{c)}{ }^{a_{1} \ldots a_{k-1}} \\
& +2 \Upsilon_{r} \delta_{(b}^{\left(a_{1}\right.} f_{c)}^{\left.a_{2} \ldots a_{k-1}\right)_{0} r} \\
& \widehat{\nabla}_{(b} f_{c)}{ }^{a_{1} \ldots a_{k-1}}=\nabla_{(b} f_{c)}{ }^{a_{1} \ldots a_{k-1}}+(k-1) \Upsilon_{(b} f_{c)}{ }^{a_{1} \ldots a_{k-1}}-(k-1) \Upsilon^{\left(a_{1}\right.} f^{\left.a_{2} \ldots a_{k-1}\right)} b c \\
& +\mathrm{g}_{b c} \Upsilon_{r} f^{a_{1} \ldots a_{k-1} r}+(k-1) \Upsilon_{r} \delta_{(b}^{\left(a_{1}\right.} f_{c)} a_{\left.2 \ldots a_{k-1}\right) r} .
\end{aligned}
$$

We are interested in linear combinations of terms in (3.9) and (3.10) which are independent on the rescaling $\hat{g}=e^{2 \Upsilon}$ g. Considering formulas (3.12)-(3.17), we observe that the term $\nabla_{(a} \nabla_{b} \Upsilon_{c)_{0}}$ appears only in (3.14) and the term $\nabla_{a} \nabla^{r} \Upsilon_{r}$ appears only on the right hand side of (3.15). This means, terms $\left(\nabla_{r} \mathrm{~J}\right) f^{a_{1} \ldots a_{k-1} r}$ and $\left(\nabla_{(r} \mathrm{P}_{s t)_{0}}\right) \mathrm{g}^{t\left(a_{1}\right.} f^{\left.a_{2} \ldots a_{k-1}\right)_{0} r s}$ do not appear in the required linear combination.

The Weyl tensor appears in the conformal transformation of the terms in (3.9) but not of the ones in (3.10). Therefore, we look for conformally invariant linear combinations

$$
\begin{aligned}
& x_{1} \mathrm{C}^{r}{ }_{s t}{ }^{\left(a_{1}\right.} \nabla_{r} f^{\left.a_{2} \ldots a_{k-1}\right)_{0} s t}+x_{2} \mathrm{C}_{r}^{\left(a_{1}{ }_{r}{ }_{2}{ }_{s} \nabla_{t} f^{\left.a_{3} \ldots a_{k-1}\right)_{0} r s t}\right.} \\
& \quad+x_{3}\left(\nabla_{r} \mathrm{C}_{s}{ }^{\left(a_{1}\right.}{ }_{t}{ }^{a_{2}}\right) f^{\left.a_{3} \ldots a_{k-1}\right)_{0} r s t}+x_{4} \mathrm{~A}_{r s}{ }^{\left(a_{1}\right.} f^{\left.a_{2} \ldots a_{k-1}\right)_{0} r s}
\end{aligned}
$$


and

$$
\begin{aligned}
& y_{1} \mathrm{~J} \nabla_{r} f^{a_{1} \ldots a_{k-1} r}+y_{2} \overline{\mathrm{P}}_{r s} \nabla^{(r} f^{s) a_{1} \ldots a_{k-1}} \\
& \quad+y_{3} \overline{\mathrm{P}}_{r}^{\left(a_{1}\right.} \nabla_{s} f^{\left.a_{2} \ldots a_{k-1}\right)_{0} r s}+y_{4} \overline{\mathrm{P}}_{r s} \nabla^{\left(a_{1}\right.} f^{\left.a_{2} \ldots a_{k-1}\right)_{0} r s},
\end{aligned}
$$

where $\overline{\mathrm{P}}_{r s}=\mathrm{P}_{(r s)_{0}}$ denotes the trace-free part of $\mathrm{P}$. In other words, we search for scalars $x_{i}, y_{j} \in \mathbb{R}$ such that both (3.18) and (3.19) are invariant independently.

First we discuss (3.18) which is possible only for $k \geq 2$ and some terms only for $k \geq 3$. Assuming $k \geq 3$, conformal transformations of these terms are

$$
\begin{aligned}
& \mathrm{C}^{r}{ }_{s t}\left(a_{1} \widehat{\nabla}_{r} f^{\left.a_{2} \ldots a_{k-1}\right)_{0} s t}=\mathrm{C}^{r}{ }_{s t}^{\left(a_{1}\right.} \widehat{\nabla}_{r} f^{\left.a_{2} \ldots a_{k-1}\right)_{0} s t}+(k+1) \Upsilon_{r} \mathrm{C}^{r}{ }_{s t}{ }^{\left(a_{1}\right.} f^{\left.a_{2} \ldots a_{k-1}\right)_{0} s t}\right. \\
& +(k-2) \mathrm{C}^{\left(a_{1}\right.}{ }_{s t}^{a_{2}} \Upsilon_{r} f^{\left.a_{3} \ldots a_{k-1}\right)_{0} s t r},
\end{aligned}
$$

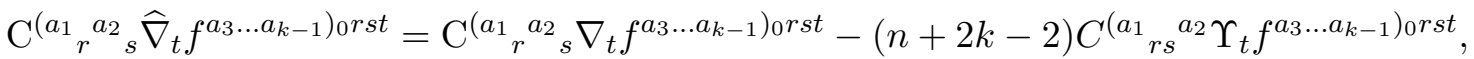

$$
\begin{aligned}
& \left(\widehat{\nabla}_{r} \mathrm{C}_{s}{ }^{\left(a_{1}\right.}{ }_{t}{ }^{a_{2}}\right) f^{\left.a_{3} \ldots a_{k-1}\right)_{0}{ }^{r s t}}=\left(\nabla_{r} \mathrm{C}_{s}{ }^{\left(a_{1}\right.} t_{t}^{a_{2}}\right) f^{\left.a_{3} \ldots a_{k-1}\right)_{0} r s t}+4 \mathrm{C}^{\left(a_{1}\right.}{ }_{r s}{ }^{a_{2}} \Upsilon_{t} f^{\left.a_{3} \ldots a_{k-1}\right)_{0} r s t} \\
& -2 \Upsilon_{r} \mathrm{C}^{r}{ }_{s t}{ }^{\left(a_{1}\right.} f^{\left.a_{2} \ldots a_{k-1}\right)_{0} s t} \text {, } \\
& \widehat{\mathrm{A}}_{r s}{ }^{\left(a_{1}\right.} f^{\left.a_{2} \ldots a_{k-1}\right)_{0} r s}=\mathrm{A}_{r s}{ }^{\left(a_{1}\right.} f^{\left.a_{2} \ldots a_{k-1}\right)_{0} r s}+\Upsilon_{r} \mathrm{C}^{r}{ }_{s t}{ }^{\left(a_{1}\right.} f^{\left.a_{2} \ldots a_{k-1}\right)_{0} s t}
\end{aligned}
$$

using (3.17), (3.16) and (3.13). Now, considering where the term $\Upsilon_{r} \mathrm{C}^{r} s t^{\left(a_{1}\right.} f^{\left.a_{2} \ldots a_{k-1}\right)_{0} s t}$ appears in the previous display, we see that $(k+1) x_{1}-2 x_{3}+x_{4}=0$. Considering the other term $\mathrm{C}^{\left(a_{1}\right.}{ }_{r s}{ }^{a_{2}} \Upsilon_{t} f^{\left.a_{3} \ldots a_{k-1}\right)_{0} r s t}$, we conclude that $(k-2) x_{1}-(n+2 k-2) x_{2}+4 x_{3}=0$. Solutions of this pair of linear equations are generated by $\left(x_{1}, x_{2}, x_{3}, x_{4}\right)=(n+2 k-2, k-2,0,-(k+1)(n+2 k-2))$ and $\left(x_{1}, x_{2}, x_{3}, x_{4}\right)=(0,4, n+2 k-2,2(n+2 k-2))$, therefore the space of corresponding invariant linear operators is generated by the operators $\mathbf{F}_{1}$ and $\mathbf{F}_{2}$ defined in the following way:

$$
\begin{aligned}
& \left(\mathbf{F}_{1}(f)\right)^{a_{1} \ldots a_{k-1}}=\mathrm{C}^{r}{ }_{s t}{ }^{\left(a_{1}\right.} \nabla_{r} f^{\left.a_{2} \ldots a_{k-1}\right)_{0} s t}-(k+1) \mathrm{A}_{r s}{ }^{\left(a_{1}\right.} f^{\left.a_{2} \ldots a_{k-1}\right)_{0} r s}
\end{aligned}
$$

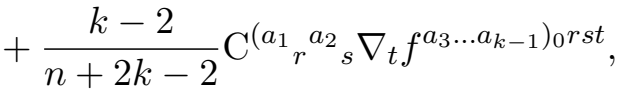

$$
\begin{aligned}
& \left(\mathbf{F}_{2}(f)\right)^{a_{1} \ldots a_{k-1}}=4 \mathrm{C}^{\left(a_{1}\right.}{ }_{r}^{a_{2}}{ }_{s} \nabla_{t} f^{\left.a_{3} \ldots a_{k-1}\right)_{0} r s t}+(n+2 k-2)\left(\nabla_{r} \mathrm{C}_{s}{ }^{\left(a_{1}\right.}{ }_{t}{ }^{a_{2}}\right) f^{\left.a_{3} \ldots a_{k-1}\right)_{0} r s t} \\
& +2(n+2 k-2) \mathrm{A}_{r s}{ }^{\left(a_{1}\right.} f^{\left.a_{2} \ldots a_{k-1}\right)_{0} r s} \text {. }
\end{aligned}
$$

This shows that the operators in the statement of the proposition for $k \geq 3$ are invariant.

In the case $k=2$ only some terms from (3.18) can appear. Specifically, we study the conformal invariance of the linear combination

$$
x_{1} \mathrm{C}^{r}{ }_{s t}^{a} \nabla_{r} f^{s t}+x_{4} \mathrm{~A}_{r s}{ }^{a} f^{r s}
$$

for the section $f^{a b}$ in $\Gamma\left(S^{2,0} T M\right)$. Since $\widehat{\nabla}_{a} f^{b c}=\nabla_{a} f^{b c}+2 \Upsilon_{a} f^{b c}-2 \Upsilon^{(b} f^{c)}{ }_{a}+2 \delta_{a}{ }^{(b} \Upsilon_{r} f^{c) r}$, conformal transformations of terms in the previous display are

$$
\begin{aligned}
& \mathrm{C}^{r}{ }_{s t}{ }^{a} \widehat{\nabla}_{r} f^{s t}=\mathrm{C}^{r}{ }_{s t}{ }^{a} \widehat{\nabla}_{r} f^{s t}+3 \Upsilon_{r} \mathrm{C}^{r}{ }_{s t}^{a} f^{s t} \quad \text { and } \\
& \widehat{\mathrm{A}}_{r s}{ }^{a} f^{r s}=\mathrm{A}_{r s}{ }^{a} f^{r s}+\Upsilon_{r} \mathrm{C}^{r}{ }_{s t}{ }^{a} f^{s t} .
\end{aligned}
$$

By the same reasoning as in the case $k \geq 3$, we obtain that the operator given in (3.20) is invariant if and only if $\left(x_{1}, x_{4}\right)$ is a multiple of $(1,-3)$. In the case $k=2$, the only invariant operators are thus the multiples of the operator $\mathbf{F}$ defined by

$$
(\mathbf{F}(f))^{a}=\mathrm{C}^{r}{ }_{s t}^{a} \nabla_{r} f^{s t}-3 \mathrm{~A}_{r s}{ }^{a} f^{r s} .
$$

Now we shall discuss terms (3.19) and we assume $k \geq 2$ first. Consider an arbitrary but fixed point $x \in M$. We can choose the function $\Upsilon$ such that $\Upsilon_{a}(x)=0, \nabla_{(a} \Upsilon_{b)_{0}}(x)=\Phi_{a b}(x)$ 
and $\nabla^{r} \Upsilon_{r}(x)=\Psi(x)$ for any prescribed values of $\Phi_{a b}(x)$ and $\Psi(x)$. Therefore, the conformal transformation of terms in (3.19) is

$$
\begin{aligned}
& \widehat{\mathrm{J}} \widehat{\nabla}_{r} f^{a_{1} \ldots a_{k-1} r}=\mathrm{J} \nabla_{r} f^{a_{1} \ldots a_{k-1} r}-\Psi \nabla_{r} f^{a_{1} \ldots a_{k-1} r}, \\
& \widehat{\overline{\mathrm{P}}}_{r s} \widehat{\nabla}^{(r} f^{s) a_{1} \ldots a_{k-1}}=\overline{\mathrm{P}}_{r s} \nabla^{(r} f^{s) a_{1} \ldots a_{k-1}}-\Phi_{r s} \nabla^{(r} f^{s) a_{1} \ldots a_{k-1},} \\
& \widehat{\overline{\mathrm{P}}}_{r}^{\left(a_{1}\right.} \widehat{\nabla}_{s} f^{\left.a_{2} \ldots a_{k-1}\right)_{0} r s}=\overline{\mathrm{P}}_{r}{ }^{\left(a_{1}\right.} \nabla_{s} f^{\left.a_{2} \ldots a_{k-1}\right)_{0} r s}-\Phi_{r}{ }^{\left(a_{1}\right.} \nabla_{s} f^{\left.a_{2} \ldots a_{k-1}\right)_{0} r s}, \\
& \widehat{\overline{\mathrm{P}}}_{r s} \widehat{\nabla}^{\left(a_{1}\right.} f^{\left.a_{2} \ldots a_{k-1}\right)_{0} r s}=\overline{\mathrm{P}}_{r s} \nabla^{\left(a_{1}\right.} f^{\left.a_{2} \ldots a_{k-1}\right)_{0} r s}-\Phi_{r s} \nabla^{\left(a_{1}\right.} f^{\left.a_{2} \ldots a_{k-1}\right)_{0} r s}
\end{aligned}
$$

at the point $x$ (which is for simplicity omitted in the previous display). Choosing $\Psi(x) \neq 0$ and $\Phi_{r s}(x)=0$, the invariance of (3.19) means that $y_{1}=0$. Henceforth we assume $\Psi(x)=0$ and $\Phi_{r s}(x) \neq 0$. To determine $y_{2}, y_{3}$ and $y_{4}$, we shall test invariance of (3.19) for $f^{a_{1} \ldots a_{k}}$ with specific properties at $x$. First assume that $\nabla^{b} f^{a_{1} \ldots a_{k}}(x)=\nabla^{(b} f^{\left.a_{1} \ldots a_{k}\right)}(x)$, or equivalently that $\nabla^{b} f^{a_{1} \ldots a_{k}}(x)=\nabla^{a_{1}} f^{b a_{2} \ldots a_{k}}(x)$. This in particular implies that $\nabla_{r} f^{a_{1} \ldots a_{k-1} r}(x)=0$ and the invariance of (3.19) then means that $y_{2}+y_{4}=0$. Second, we assume $\nabla^{(b} f^{\left.a_{1} \ldots a_{k}\right)}(x)=0$ or equivalently $2 \nabla^{(b} f^{c) a_{1} \ldots a_{k-1}}(x)+(k-1) \nabla^{\left(a_{1}\right.} f^{\left.a_{2} \ldots a_{k-1}\right) b c}(x)=0$. This also implies $\nabla_{r} f^{a_{1} \ldots a_{k-1} r}(x)=0$ and the invariance of (3.19) now means that $-\frac{k-1}{2} y_{2}+y_{4}=0$. Overall, this yields $y_{2}=y_{4}=0$, and $y_{3}=0$ follows. All scalars in (3.19) are thus equal to zero. This completes the proof of the part (ii) of the proposition.

If $k=1$, (3.19) reduces to the linear combination $y_{1} \mathrm{~J} \nabla_{r} f^{r a}+y_{2} \overline{\mathrm{P}}_{r s} \nabla^{r} f^{s a}$. As above, the choice $\Psi(x) \neq 0$ and $\Phi_{r s}(x)=0$ shows that $y_{1}=0$. Hence $y_{2}=0$ and the part $(i)$ follows.

In order to complete the proof of the part (iii), it remains to describe natural and conformally invariant operators $\Gamma\left(S^{k, 0} T M\right) \rightarrow \Gamma\left(S^{k-3} T M\right)$. The space of these operators is also trivial in the flat case $[4,5]$, hence the first term in (3.11) cannot appear. Thus the required operator is a linear combination of the form

$$
x_{1} \mathrm{P}_{(r s)_{0}} \nabla_{t} f^{a_{1} \ldots a_{k-3} r s t}+x_{2}\left(\nabla_{r} \mathrm{P}_{s t}\right) f^{a_{1} \ldots a_{k-3} r s t},
$$

where $x_{1}, y_{1} \in \mathbb{R}$. Reasoning similarly as above, we observe that $\nabla_{(a} \nabla_{b} \Upsilon_{c)_{0}}$ appears only in the conformal transformation of the second term in the previous display. Therefore $x_{2}=0$, hence also $x_{1}=0$ and the proposition follows.

\section{Classification of second order symmetries of $\Delta_{Y}$}

We start this section with the definition of the algebra $\mathcal{A}$ of conformal symmetries of the conformal Laplacian. Afterwards, we provide our main result: a complete description of the space $\mathcal{A}^{2}$ of second order conformal symmetries.

\subsection{The algebra of symmetries of the conformal Laplacian}

Let $(M,[\mathrm{~g}])$ be a conformal manifold of dimension $n$. Fixing a metric $\mathrm{g} \in[\mathrm{g}]$, we can regard the conformal Laplacian, $\Delta_{Y}=\nabla_{a} \mathrm{~g}^{a b} \nabla_{b}-\frac{n-2}{4(n-1)} \mathrm{Sc}$, as acting on functions. The symmetries of $\Delta_{Y}$ are defined as differential operators which commute with $\Delta_{Y}$. Hence, they preserve the eigenspaces of $\Delta_{Y}$. More generally, conformal symmetries $D_{1}$ are defined by the weaker algebraic condition

$$
\Delta_{Y} \circ D_{1}=D_{2} \circ \Delta_{Y}
$$

for some differential operator $D_{2}$, so that they only preserve the kernel of $\Delta_{Y}$. The operator $\Delta_{Y}$ can be considered in equation (4.1) as acting between different line bundles and in particular as an element of $\mathcal{D}_{\lambda_{0}, \mu_{0}}$, where $\lambda_{0}=\frac{n-2}{2 n}, \mu_{0}=\frac{n+2}{2 n}$. With this choice, $\Delta_{Y}$ is conformally invariant 
and the space of conformal symmetries depends only on the conformal class of the metric g. It is stable under linear combinations and compositions.

The operators of the form $P \Delta_{Y}$, i.e. in the left ideal generated by $\Delta_{Y}$, are obviously conformal symmetries. Since they act trivially on the kernel of $\Delta_{Y}$, they are considered as trivial. Following [15, 18, 26], this leads to

Definition 4.1. Let $(M,[\mathrm{~g}])$ be a conformal manifold with conformal Laplacian $\Delta_{Y} \in \mathcal{D}_{\lambda_{0}, \mu_{0}}$. The algebra of conformal symmetries of $\Delta_{Y}$ is defined as

$$
\mathcal{A}:=\left\{D_{1} \in \mathcal{D}_{\lambda_{0}, \lambda_{0}} \mid \exists D_{2} \in \mathcal{D}_{\mu_{0}, \mu_{0}} \text { s.t. } D_{2} \circ \Delta_{Y}=\Delta_{Y} \circ D_{1}\right\},
$$

and the subspace of trivial symmetries as

$$
\left(\Delta_{Y}\right):=\left\{A \Delta_{Y} \mid A \in \mathcal{D}_{\mu_{0}, \lambda_{0}}\right\}
$$

Thus, $\mathcal{A}$ is a subalgebra of $\mathcal{D}_{\lambda_{0}, \lambda_{0}}$ and $\left(\Delta_{Y}\right)$ is the left ideal generated by $\Delta_{Y}$ in $\mathcal{D}_{\lambda_{0}, \lambda_{0}}$. The filtration by the order on $\mathcal{D}_{\lambda_{0}, \lambda_{0}}$ induces a filtration on $\mathcal{A}$ and we denote by

$$
\mathcal{A}^{k}:=\mathcal{A} \cap \mathcal{D}_{\lambda_{0}, \lambda_{0}}^{k}
$$

the algebra of conformal symmetries of order $k$. Obviously, $\mathcal{A}^{0} \simeq \mathbb{R}$ is the space of constant functions, identified with zero order operators on $\lambda_{0}$-densities. Moreover, the invariance of $\Delta_{Y}$ under the action of conformal Killing vector fields, see (2.9), shows that $\mathcal{A}^{1}$ is the direct sum of $\mathcal{A}^{0}$ with the space of Lie derivatives $L_{X}^{\lambda_{0}} \in \mathcal{D}_{\lambda_{0}, \lambda_{0}}^{1}$ along conformal Killing vector fields $X$. Since $\mathcal{A}$ is an algebra, $\mathcal{A}^{2}$ contains in particular $L_{X}^{\lambda_{0}} \circ L_{Y}^{\lambda_{0}}$ for $X, Y$ conformal Killing vector fields.

\subsection{The algebra of symmetries of the null geodesic flow}

Let $(M, \mathrm{~g})$ be a pseudo-Riemannian manifold and $\left(x^{i}, p_{i}\right)$ denote a canonical coordinate system on $T^{*} M$. The inverse metric $\mathrm{g}^{-1}$ pertains to $\Gamma\left(S^{2} T M\right)$ and identifies with $H:=\mathrm{g}^{i j} p_{i} p_{j} \in \mathcal{S}_{0}$, where $\mathcal{S}_{0}=\operatorname{Pol}\left(T^{*} M\right) \cong \Gamma(S T M)$ (see Section 2.1). Along the isomorphism $T^{*} M \cong T M$ provided by the metric, the Hamiltonian flow of $H$ corresponds to the geodesic flow of $\mathrm{g}$.

The symmetries of the geodesic flow are given by functions $K \in \mathcal{S}_{0}$ which Poisson commute with $H$. They coincide with the symmetric Killing tensors. The null geodesic flow, i.e. the geodesic flow restricted to the level set $H=0$, depends only on the conformal class of g. It admits additional symmetries, namely all the functions $K \in \mathcal{S}_{0}$ such that

$$
\{H, K\} \in(H),
$$

where $\{\cdot, \cdot\}$ stands for the canonical Poisson bracket on $T^{*} M$, defined in $(2.3)$, and $(H)$ for the ideal spanned by $H$ in $\mathcal{S}_{0}$. The linearity and Leibniz property of the Poisson bracket ensure that the space of symmetries of the null geodesic flow is a subalgebra of $\mathcal{S}_{0}$. Besides, remark that all the functions in $(H)$ are symmetries which act trivially on the null geodesic flow.

Definition 4.2. Let $(M, \mathrm{~g})$ be a pseudo-Riemannian manifold and $H \in \mathcal{S}_{0}$ the function associated to $g$. The algebra of symmetries of the null geodesic flow of $g$ is given by the following subalgebra of $\mathcal{S}_{0}$,

$$
\mathcal{K}:=\left\{K \in \mathcal{S}_{0} \mid\{H, K\} \in(H)\right\} .
$$


In particular, the algebra $\mathcal{K}$ contains the ideal $(H)$ of trivial symmetries. It inherits the gradation of $\mathcal{S}_{0}$ by the degree,

$$
\mathcal{K}^{k}:=\mathcal{K} \cap \mathcal{S}_{0}^{k}
$$

The space $\mathcal{K}^{0}$ is the space of constant functions on $T^{*} M$. The Hamiltonian flows of functions in $\mathcal{K}^{1}$ coincide with the Hamiltonian lift to $T^{*} M$ of the conformal Killing vectors on $(M$, $[\mathrm{g}])$. For higher degrees, the elements in $\mathcal{K}$ are symmetric conformal Killing tensors whose Hamiltonian flows do not preserve the configuration manifold $M$. They are symmetries of the whole phase space but not of the configuration manifold and often named hidden symmetries by physicists.

Proposition 4.3. The elements $K \in \mathcal{K}^{k}$ are symmetric conformal Killing $k$-tensors. They are characterized equivalently as:

- symmetric tensors of order $k$ s.t. $\nabla_{\left(a_{0}\right.} K_{\left.a_{1} \ldots a_{k}\right)_{0}}=0$,

- symbols of degree $k$ satisfying $\{H, K\} \in(H)$,

- elements of $\mathcal{S}_{0}^{k}$ in the kernel of the conformal Killing operator $\mathbf{G}$ (see (3.5) or (3.6)).

The proof is both classical and straightforward, we let it to the reader. The next proposition is essential to determine the algebra $\mathcal{A}$ of conformal symmetries.

Proposition 4.4. If $D_{1} \in \mathcal{A}^{k}$ then $\sigma_{k}\left(D_{1}\right) \in \mathcal{K}^{k}$. Under the identification $\operatorname{gr} \mathcal{D}_{\lambda_{0}, \lambda_{0}} \cong \mathcal{S}_{0}$, the associated graded algebra gr $\mathcal{A}$ becomes a subalgebra of $\mathcal{K}$ and $\operatorname{gr}\left(\Delta_{Y}\right)$ identifies with $(H)$.

Proof. Suppose that $D_{1}$ is a conformal symmetry of order $k$, i.e. satisfies $\Delta_{Y} \circ D_{1}=D_{2} \circ \Delta_{Y}$ for some $D_{2}$. Working in the algebra $\mathcal{D}_{\lambda_{0}, \lambda_{0}}$ we deduce that $\left[\Delta_{Y}, D_{1}\right] \in\left(\Delta_{Y}\right)$ and the property $(2.5)$ leads then to $\left\{H, \sigma_{k}\left(D_{1}\right)\right\} \in(H)$, i.e. $\sigma_{k}\left(D_{1}\right) \in \mathcal{K}^{k}$. The inclusion gr $\mathcal{A} \leq \mathcal{K}$ follows. As $\sigma_{2}\left(\Delta_{Y}\right)=H$, the property (2.4) of the principal symbol maps implies that $\operatorname{gr}\left(\Delta_{Y}\right) \cong(H)$.

\subsection{Second order conformal symmetries}

We adapt the strategy used in [26], dealing with conformally flat manifolds. Thanks to a natural and conformally invariant quantization, we get a first description of the potential obstruction for a conformal Killing tensor giving rise to a conformal symmetry of $\Delta_{Y}$.

Theorem 4.5. Let $\mathcal{Q}_{\lambda, \mu}$ be a family of natural and conformally invariant quantizations as in Theorem 2.5. We get then

$$
\Delta_{Y} \circ \mathcal{Q}_{\lambda_{0}, \lambda_{0}}(S)-\mathcal{Q}_{\mu_{0}, \mu_{0}}(S) \circ \Delta_{Y}=\mathcal{Q}_{\lambda_{0}, \mu_{0}}(2 \mathbf{G}(S)+\operatorname{Obs}(S)), \quad \forall S \in \mathcal{S}_{0}^{\leq 2} .
$$

The operator Obs is the natural and conformally invariant operator defined by

$$
\text { Obs }=\frac{2(n-2)}{3(n+1)} \mathbf{F}
$$

where $(\mathbf{F}(S))^{a}=\mathrm{C}^{r}{ }_{s t}{ }^{a} \nabla_{r} S^{s t}-3 \mathrm{~A}_{r s}{ }^{a} S^{r s}$ for $S \in \mathcal{S}_{0}^{2}$ and we set $\mathbf{F}(S)=0$ for $S \in \mathcal{S}_{0}^{\leq 1}$.

Proof. According to (2.6), we have $\mathcal{S}_{0}^{2}=\mathcal{S}_{0}^{2,0} \oplus \mathcal{S}_{0}^{2,1}$ and $S \in \mathcal{S}_{0}^{2,1}$ is of the following form $S=\left(\left|\operatorname{Vol}_{\mathrm{g}}\right|{ }^{\delta_{0}} H\right) S_{0}$ with $S_{0} \in \mathcal{F}_{-\delta_{0}}$. By Theorem 2.5, we have the identities

$$
\mathcal{Q}_{\lambda_{0}, \lambda_{0}}(S)=\mathcal{Q}_{\mu_{0}, \lambda_{0}}\left(S_{0}\right) \circ \Delta_{Y} \quad \text { and } \quad \mathcal{Q}_{\mu_{0}, \mu_{0}}(S)=\Delta_{Y} \circ \mathcal{Q}_{\mu_{0}, \lambda_{0}}\left(S_{0}\right) .
$$

Besides, from the expressions of the operators $\mathbf{G}$ and $\mathbf{F}$ (see e.g. (3.6)), we deduce

$$
\mathbf{G}(S)=0 \quad \text { and } \quad \operatorname{Obs}(S)=0 .
$$

Hence the equality (4.2) holds for all $S \in \mathcal{S}_{0}^{2,1}$. 
Next, we define a natural and conformally invariant operator QS on $\mathcal{D}_{\lambda_{0}, \lambda_{0}}^{2}$ by $D \mapsto \Delta_{Y} \circ$ $D-D \circ \Delta_{Y}$. Pulling this map back to trace-free symbols via the quantization maps,

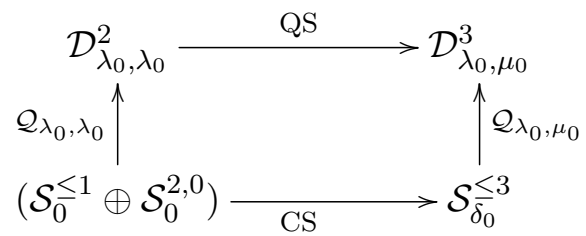

this leads to a natural and conformally invariant operator CS on $\mathcal{S}_{0}^{\leq 1} \oplus \mathcal{S}_{0}^{2,0}$. Since $\Delta_{Y}$ is formally self-adjoint and the quantization maps satisfy the reality condition (2.13), we deduce that, for all $S \in \mathcal{S}_{0}^{k, 0}$,

$$
\Delta_{Y} \circ \mathcal{Q}_{\lambda_{0}, \lambda_{0}}(S)-\mathcal{Q}_{\mu_{0}, \mu_{0}}(S) \circ \Delta_{Y}
$$

is of degree $k+1$ and is formally skew-adjoint (resp. self-adjoint) if $k$ is even (resp. odd). As such, it is of the form $\mathcal{Q}_{\lambda_{0}, \mu_{0}}(P)$, with $P \in \mathcal{S}_{\delta_{0}}^{3} \oplus \mathcal{S}_{\delta_{0}}^{1}$ if $S$ is of degree $2, P \in \mathcal{S}_{\delta_{0}}^{2} \oplus \mathcal{S}_{\delta_{0}}^{0}$ if $S$ is of degree 1 and $P \in \mathcal{S}_{\delta_{0}}^{1}$ if $S$ is of degree 0 . We can reduce accordingly the target space of CS restricted to homogeneous symbols. Applying Proposition 3.1 and Proposition 3.2, we deduce that $\mathrm{CS}=a \mathbf{G}+b \mathbf{F}$ for some real constants $a, b$. We have then

$$
\Delta_{Y} \circ \mathcal{Q}_{\lambda_{0}, \lambda_{0}}(S)-\mathcal{Q}_{\mu_{0}, \mu_{0}}(S) \circ \Delta_{Y}=\mathcal{Q}_{\lambda_{0}, \mu_{0}}(a \mathbf{G}(S)+b \mathbf{F}(S)), \quad \forall S \in \mathcal{S}_{0}^{\leq 2} .
$$

It is straightforward to prove that $a=2$. To prove $b=\frac{2(n-2)}{3(n+1)}$, we study a specific conformal symmetry of $\Delta_{Y}$.

Lemma 4.6. Let $\eta$ be the pseudo-Euclidean flat metric of signature $(p, q), h$ a non-vanishing function on $\mathbb{R}^{2}$ and $n=p+q+2$. Let $\left(M_{0}, \mathrm{~g}\right)$ be the pseudo-Riemannian manifold $\left(\mathbb{R}^{2} \times\right.$ $\left.\mathbb{R}^{n-2}, \mathrm{~g}_{0} \times \eta\right)$, where the metric on $\mathbb{R}^{2}$ is determined by $\left(\mathrm{g}_{0}\right)^{-1}=h\left(x_{1}, x_{2}\right) p_{1}^{2}+p_{2}^{2}$ in canonical Cartesian coordinates $\left(x^{i}, p_{i}\right)$ on $T^{*} \mathbb{R}^{n}$. Then, $K=p_{3}^{2}$ is a Killing tensor on $\left(M_{0}, \mathrm{~g}\right)$, and we have the following relation:

$$
\Delta_{Y} \circ \mathcal{Q}_{\lambda_{0}, \lambda_{0}}(K)-\mathcal{Q}_{\mu_{0}, \mu_{0}}(K) \circ \Delta_{Y}=\mathcal{Q}_{\lambda_{0}, \mu_{0}}(\operatorname{Obs}(K)) \neq 0 .
$$

Proof. Using the relation that links the coefficients of $\mathrm{g}$ and the Christoffel symbols $\Gamma_{j k}^{i}$ of the associated Levi-Civita connection, it is obvious that $\Gamma_{j k}^{i}=0$ if at least one of the indices $i$, $j, k$ is greater than or equal to 3 . Thus, the only non-vanishing components of the Riemann tensor and the Ricci tensor associated with $g$ are given by the corresponding components of the Riemann tensor and the Ricci tensor of $g_{0}$. In the same way, the scalar curvature of $g$ is equal to the scalar curvature of $\mathrm{g}_{0}$.

Using these facts and the formula for $\mathcal{Q}_{\lambda_{0}, \lambda_{0}}(K)$ presented in the proof of Proposition 4.9, it is easy to see that

$$
\mathcal{Q}_{\lambda_{0}, \lambda_{0}}(K)=\mathcal{Q}_{\mu_{0}, \mu_{0}}(K)=\partial_{x^{3}}^{2}+\frac{1}{2(n-1)(n+1)} \mathrm{Sc} .
$$

By a direct computation, we obtain the following relation:

$$
\begin{aligned}
\Delta_{Y} & \circ \mathcal{Q}_{\lambda_{0}, \lambda_{0}}(K)-\mathcal{Q}_{\mu_{0}, \mu_{0}}(K) \circ \Delta_{Y} \\
& =\left[\Delta_{Y}, \mathcal{Q}_{\lambda_{0}, \lambda_{0}}(K)\right]=\frac{1}{(n-1)(n+1)} \mathcal{Q}_{\lambda_{0}, \mu_{0}}\left(\mathrm{~g}^{i j}\left(\partial_{i} \mathrm{Sc}\right) p_{j}\right)+f,
\end{aligned}
$$

with $f \in \mathcal{C}^{\infty}(M)$. According to (4.3), the function $f$ vanishes. 
Besides, we can compute easily the Cotton-York tensor A associated with g. Indeed, if $\mathrm{P}$ denotes the Schouten tensor, we have

$$
\mathrm{A}_{i j k}=2 \nabla_{[i} \mathrm{P}_{j] k}=\frac{2}{n-2} \nabla_{[i}\left(\operatorname{Ric}_{j] k}-\frac{1}{2(n-1)} \mathrm{g}_{j] k} \mathrm{Sc}\right) .
$$

Using the peculiar form of $K$ and the remark done previously about the Christoffel symbols and the curvature tensors of $g$, it is obvious that

$$
\mathrm{A}_{i j k} K^{j k}=-\frac{1}{2(n-1)(n-2)} \partial_{i} \mathrm{Sc}
$$

for all $i$. The conclusion follows immediately.

By naturality of the map CS defined above, the coefficient $b$ in (4.3) depends only on the signature of the metric. As $b$ is equal to $\frac{2(n-2)}{3(n+1)}$ in the example presented in the previous lemma, where the dimension $M_{0}$ is of arbitrary dimension $n$ and $g$ of arbitrary signature, we conclude that $b=\frac{2(n-2)}{3(n+1)}$ in $(4.3)$.

Obviously, we have $\operatorname{Obs}(S)=0$ if $S$ is a symbol of degree 0 or 1 . Thus, we recover that $\mathcal{A}^{1} \cong \mathcal{K}^{1} \oplus \mathcal{K}^{0}$ and the isomorphism is provided by $\mathcal{Q}_{\lambda_{0}, \lambda_{0}}$. Since the symmetric conformal Killing tensors $K$ satisfy $\mathbf{G} K=0$, we deduce the following

Corollary 4.7. Let $(M, \mathrm{~g})$ be a pseudo-Riemannian manifold of dimension $n$ endowed with a symmetric conformal Killing 2 -tensor $K$. The operator

$$
\begin{aligned}
\mathcal{Q}_{\lambda_{0}, \lambda_{0}}(K)= & K^{a b} \nabla_{a} \nabla_{b}+\frac{n}{n+2}\left(\nabla_{a} K^{a b}\right) \nabla_{b} \\
& +\frac{n(n-2)}{4(n+2)(n+1)}\left(\nabla_{a} \nabla_{b} K^{a b}\right)-\frac{n+2}{4(n+1)} \operatorname{Ric}_{a b} K^{a b},
\end{aligned}
$$

is a conformal symmetry of $\Delta_{Y}$ if and only if $\operatorname{Obs}(K)=0$.

Proof. Indeed, the condition is obviously sufficient. Next, the condition is necessary because if $\mathcal{Q}_{\lambda_{0}, \lambda_{0}}(K)$ is a conformal symmetry of $\Delta_{Y}$, there exists a differential operator $D$ such that

$$
\Delta_{Y} \circ \mathcal{Q}_{\lambda_{0}, \lambda_{0}}(K)=D \circ \Delta_{Y}
$$

We have then successively, using Theorem 4.5:

$$
\begin{aligned}
0 & =\Delta_{Y} \circ \mathcal{Q}_{\lambda_{0}, \lambda_{0}}(K)-D \circ \Delta_{Y} \\
& =\left(\Delta_{Y} \circ \mathcal{Q}_{\lambda_{0}, \lambda_{0}}(K)-\mathcal{Q}_{\mu_{0}, \mu_{0}}(K) \circ \Delta_{Y}\right)+\left(\mathcal{Q}_{\mu_{0}, \mu_{0}}(K) \circ \Delta_{Y}-D \circ \Delta_{Y}\right) \\
& =\mathcal{Q}_{\lambda_{0}, \mu_{0}}(\operatorname{Obs}(K))+\left(\mathcal{Q}_{\mu_{0}, \mu_{0}}(K)-D\right) \Delta_{Y} .
\end{aligned}
$$

The operator $\mathcal{Q}_{\lambda_{0}, \mu_{0}}(\operatorname{Obs}(K))$ is of order one but not the operator $\left(\mathcal{Q}_{\mu_{0}, \mu_{0}}(K)-D\right) \Delta_{Y}$, unless it vanishes. Hence, both terms $\mathcal{Q}_{\lambda_{0}, \mu_{0}}(\mathbf{O b s}(K))$ and $\left(\mathcal{Q}_{\mu_{0}, \mu_{0}}(K)-D\right) \Delta_{Y}$ have to vanish and then $\operatorname{Obs}(K)=0$.

In particular, on a conformally flat manifold, all the conformal Killing 2-tensors give rise to conformal symmetries of $\Delta_{Y}$ after quantization by $\mathcal{Q}_{\lambda_{0}, \lambda_{0}}$, as proved in [26]. We are now in position to prove our main theorem, which provides a full description of the conformal symmetries of $\Delta_{Y}$ given by second order differential operators. The isomorphism $\Gamma(T M) \cong \Gamma\left(T^{*} M\right)$ provided by the metric is denoted by ${ }^{b}$. 
Theorem 4.8. The second order conformal symmetries of $\Delta_{Y}$ are classif ied as follows:

(i) $\mathcal{A}^{1}=\left\{L_{X}^{\lambda_{0}}+c \mid c \in \mathbb{R}\right.$ and $\left.X \in \mathcal{K}^{1}\right\}$,

(ii) $\mathcal{A}^{2} / \mathcal{A}^{1} \cong\left\{K \in \mathcal{K}^{2} \mid \operatorname{Obs}(K)^{b}\right.$ is an exact 1 -form $\}$, and if $K \in \mathcal{K}^{2}$ satisfies $\operatorname{Obs}(K)^{b}$ $=-2 d f$, with $f \in \mathcal{C}^{\infty}(M)$, the corresponding element in $\mathcal{A}^{2} / \mathcal{A}^{1}$ is given by

$$
\mathcal{Q}_{\lambda_{0}, \lambda_{0}}(K)+f
$$

Proof. We deduce from Proposition 4.4 that the principal symbol $K$ of a second-order conformal symmetry $D_{1}$ is a symmetric conformal Killing 2-tensor. Since quantization maps are bijective, the operator $D_{1}$ reads as

$$
D_{1}=\mathcal{Q}_{\lambda_{0}, \lambda_{0}}(K+X+f),
$$

with $f$ and $X$ symbols of degree 0 and 1 respectively. Theorem 4.5 implies that

$$
\Delta_{Y} \circ D_{1}-\mathcal{Q}_{\mu_{0}, \mu_{0}}(K+X+f) \circ \Delta_{Y}=\mathcal{Q}_{\lambda_{0}, \mu_{0}}(2 \mathbf{G}(X)+\mathbf{O b s}(K)+2 \mathbf{G}(f)) .
$$

Hence $\Delta_{Y} \circ D_{1} \in\left(\Delta_{Y}\right)$ leads to $\mathbf{G}(X) \in(H)$. By definition of $\mathbf{G}$, this means that $\mathbf{G}(X)=0$, i.e. $X \in \mathcal{K}^{1}$. As the symbols $\operatorname{Obs}(K)$ and $\mathbf{G}(f)$ are of degree 1 , they cannot pertains to $\left(\Delta_{Y}\right)$. Therefore, $\Delta_{Y} \circ D_{1} \in\left(\Delta_{Y}\right)$ is equivalent to $X \in \mathcal{K}^{1}$ and $\operatorname{Obs}(K)+2 \mathbf{G}(f)=0$.

The items $(i)$ and $(i i)$ in the statement of the theorem are then easily proved.

\subsection{Second order symmetries}

The general formula (2.11) for the natural and conformally invariant quantization on symbols of degree 2 leads to the following result.

Proposition 4.9. Let $(M, \mathrm{~g})$ be a pseudo-Riemannian manifold of dimension $n$ endowed with a symmetric Killing 2 -tensor $K$. The operator

$$
\begin{aligned}
\mathcal{Q}_{\lambda_{0}, \lambda_{0}}(K)= & \mathcal{Q}_{\mu_{0}, \mu_{0}}(K)=K^{a b} \nabla_{a} \nabla_{b}+\left(\nabla_{a} K^{a b}\right) \nabla_{b}-\frac{n-2}{4(n+1)}\left(\nabla_{a} \nabla_{b} K^{a b}\right) \\
& -\frac{n+2}{4(n+1)} \operatorname{Ric}_{a b} K^{a b}+\frac{1}{2(n-1)(n+1)} \operatorname{Sc}\left(g_{a b} K^{a b}\right),
\end{aligned}
$$

is a symmetry of $\Delta_{Y}$, i.e. $\left[\Delta_{Y}, \mathcal{Q}_{\lambda_{0}, \lambda_{0}}(K)\right]=0$, if and only if $\operatorname{Obs}(K)=0$.

Proof. Let $\left(x^{i}, p_{i}\right)$ be a canonical coordinate system on $T^{*} M$. The Killing equation satisfied by $K$ reads as $\mathrm{g}^{i j} p_{i} \nabla_{j} K=0$. Applying the trace operator $\operatorname{Tr}=\mathrm{g}_{i j} \partial_{p_{i}} \partial_{p_{j}}$ we deduce that

$$
\mathrm{g}^{k l}\left(\nabla_{k} \operatorname{Tr} K\right) \nabla_{l}=-2\left(\nabla_{i} K^{i l}\right) \nabla_{l} \quad \text { and } \quad \mathrm{g}^{k l}\left(\nabla_{k} \nabla_{l} \operatorname{Tr} K\right)=-2 \nabla_{i} \nabla_{l} K^{i l} .
$$

Moreover, if $\lambda=\mu$ and $\delta=0$, we have $\beta_{1}-2 \beta_{2}=1$ and $\beta_{3}-2 \beta_{4}=\frac{n^{2} \lambda(1-\lambda)}{(n+1)(n+2)}$, where the $\beta_{i}$ are defined in (2.12). The formula for the quantization $\mathcal{Q}_{\lambda, \lambda}$ reduces then, for $K$ a Killing tensor, to

$$
\begin{aligned}
\mathcal{Q}_{\lambda, \lambda}(K)= & K^{a b} \nabla_{a} \nabla_{b}+\left(\nabla_{a} K^{a b}\right) \nabla_{b}-\frac{n^{2} \lambda(1-\lambda)}{(n+1)(n+2)}\left(\nabla_{a} \nabla_{b} K^{a b}\right) \\
& -\frac{n^{2} \lambda(\lambda-1)}{(n-2)(n+1)} \operatorname{Ric}_{a b} K^{a b}+\frac{2 n^{2} \lambda(1-\lambda)}{(n-2)(n-1)(n+1)(n+2)} \operatorname{Sc}\left(\mathrm{g}_{a b} K^{a b}\right) .
\end{aligned}
$$

Since $\lambda_{0}+\mu_{0}=1$ we deduce that $\mathcal{Q}_{\lambda_{0}, \lambda_{0}}(K)=\mathcal{Q}_{\mu_{0}, \mu_{0}}(K)$. In consequence, the equality $\left[\Delta_{Y}, \mathcal{Q}_{\lambda_{0}, \lambda_{0}}(K)\right]=0$ is equivalent to the fact that $\mathcal{Q}_{\lambda_{0}, \lambda_{0}}(K)$ is a conformal symmetry of $\Delta_{Y}$. By Corollary 4.7, this means that $\operatorname{Obs}(K)=0$. 
As a straightforward consequence, we get

Corollary 4.10. Let $(M, \mathrm{~g})$ be a conformally flat manifold and $K$ be a Killing 2-tensor. Then, we have $\left[\mathcal{Q}_{\lambda_{0}, \lambda_{0}}(K), \Delta_{Y}\right]=0$.

This corollary enlights some of the results obtained in [2]. As for conformal symmetries, we provide a full description of the symmetries of $\Delta_{Y}$ given by second order differential operators.

Theorem 4.11. The second order symmetries of $\Delta_{Y}$ are exactly the operators

$$
\mathcal{Q}_{\lambda_{0}, \lambda_{0}}(K+X)+f,
$$

where $X$ is a Killing vector field, $K$ is a Killing 2-tensor such that $\operatorname{Obs}(K)^{b}$ is an exact one-form and $f \in \mathcal{C}^{\infty}(M)$ is defined up to a constant by $\operatorname{Obs}(K)^{b}=-2 d f$.

Proof. Let $D_{1}$ be a second order symmetry of $\Delta_{Y}$. In view of (2.5), we can deduce from $\left[\Delta_{Y}, D_{1}\right]=0$ that $\left\{H, \sigma_{2}\left(D_{1}\right)\right\}=0$. This means that $K=\sigma_{2}\left(D_{1}\right)$ has to be a symmetric Killing 2 -tensor. Since quantization maps are bijective, the operator $D_{1}$ reads as

$$
D_{1}=\mathcal{Q}_{\lambda_{0}, \lambda_{0}}(K+X+f),
$$

with $f$ and $X$ symbols of degree 0 and 1 respectively. Theorem 4.5 implies that

$$
\begin{aligned}
{\left[\Delta_{Y}, D_{1}\right]=} & \mathcal{Q}_{\lambda_{0}, \mu_{0}}(2 \mathbf{G}(X)+\operatorname{Obs}(K)+2 \mathbf{G}(f)) \\
& +\left(\mathcal{Q}_{\mu_{0}, \mu_{0}}(K+X+f)-\mathcal{Q}_{\lambda_{0}, \lambda_{0}}(K+X+f)\right) \circ \Delta_{Y} .
\end{aligned}
$$

We have shown that $\mathcal{Q}_{\mu_{0}, \mu_{0}}(K)=\mathcal{Q}_{\lambda_{0}, \lambda_{0}}(K)$ in Proposition 4.9. Moreover, the general formulas in Theorem 2.4 prove that $\mathcal{Q}_{\mu_{0}, \mu_{0}}(f)=\mathcal{Q}_{\lambda_{0}, \lambda_{0}}(f)$ and $\mathcal{Q}_{\mu_{0}, \mu_{0}}(X)-\mathcal{Q}_{\lambda_{0}, \lambda_{0}}(X)=\frac{2}{n} \nabla_{a} X^{a}$. Hence, we get

$$
\left[\Delta_{Y}, D_{1}\right]=\mathcal{Q}_{\lambda_{0}, \mu_{0}}(2 \mathbf{G}(X))+\frac{2}{n}\left(\nabla_{a} X^{a}\right) \Delta_{Y}+\mathcal{Q}_{\lambda_{0}, \mu_{0}}(\mathbf{O b s}(K)+2 \mathbf{G}(f))
$$

and

$$
\sigma_{2}\left(\left[\Delta_{Y}, D_{1}\right]\right)=2 \mathbf{G}(X)+\frac{2}{n}\left(\nabla_{a} X^{a}\right) H .
$$

As $S^{2} T M=S^{2,0} T M \oplus S^{2,1} T M$, each of the two terms in the right hand side of the second equation are independent. Therefore, $\left[\Delta_{Y}, D_{1}\right]=0$ is equivalent to $\mathbf{G}(X)=0, \nabla_{a} X^{a}=0$ and $\operatorname{Obs}(K)+2 \mathbf{G}(f)=0$. The equations $\mathbf{G}(X)=0$ and $\nabla_{a} X^{a}=0$ mean that $X$ is a conformal Killing vector field with vanishing divergence, i.e. $X$ is a Killing vector field. Applying the metric, the equation $\mathbf{O b s}(K)+2 \mathbf{G}(f)=0$ translates into $\mathbf{O b s}(K)^{b}=-2 d f$. The result follows.

For comparison, we recall the alternative classification obtained in [3].

Theorem 4.12 ([3]). Let $K$ be a Killing 2-tensor and put $\mathbf{I}(K)^{a b}=K^{a c} \operatorname{Ric}_{c}^{b}-\operatorname{Ric}^{a c} K_{c}^{b}$. Then, we have

$$
\left[\nabla_{a} K^{a b} \nabla_{b}+f, \Delta+V\right]=0 \Longleftrightarrow K^{a b}\left(\nabla_{a} V\right)-\frac{1}{3}\left(\nabla_{b} \mathbf{I}(K)^{a b}\right)=\nabla^{a} f
$$

where $\Delta=\nabla_{a} \mathrm{~g}^{a b} \nabla_{b}$ and $f, V \in \mathcal{C}^{\infty}(M)$.

As an advantage of our method, the obtained condition to get a symmetry (namely $\mathbf{O b s}(K)^{b}$ exact one-form) is conformally invariant and obviously vanishes on conformally flat manifolds. As an advantage of the approach used in [3] and initiated by Carter [11], one recovers easily that

$$
\left[\Delta_{Y}, \nabla_{a} K^{a b} \nabla_{b}\right]=0
$$

for all Killing 2-tensors $K$ on an Einstein manifold. 


\subsection{Higher order conformal symmetries}

Up to now we discussed symbols of order $\leq 2$. The more general version (which we shall state without proof) of Theorem 4.5 is as follows. Assume that $\mathcal{Q}_{\lambda, \mu}$ is a family of natural and conformally invariant quantizations as in Theorem 2.5 and let $S$ be a trace-free symbol $S \in \mathcal{S}_{0}^{k, 0}$. Then we get

$$
\Delta_{Y} \circ \mathcal{Q}_{\lambda_{0}, \lambda_{0}}(S)-\mathcal{Q}_{\mu_{0}, \mu_{0}}(S) \circ \Delta_{Y}=\mathcal{Q}_{\lambda_{0}, \mu_{0}}\left(2 \mathbf{G}(S)+x \mathbf{F}_{1}(S)+y \mathbf{F}_{2}(S)+\Phi(S)\right),
$$

where operators $\mathbf{F}_{1}, \mathbf{F}_{2}: \mathcal{S}_{0}^{k, 0} \rightarrow \mathcal{S}_{\delta_{0}}^{k-1,0}$ are defined in Proposition 3.2, scalars $x$ and $y$ have the value

$$
x=\frac{k(k-1)(n+2 k-6)}{3(n+2 k-2)(n+2 k-3)} \quad \text { and } \quad y=\frac{k(k-1)(k-2)(n+2 k)}{12(n+2 k-2)(n+2 k-3)},
$$

and $\Phi$ is a natural and conformally invariant operator $\Phi: \mathcal{S}_{0}^{k, 0} \rightarrow \mathcal{S}_{\delta_{0}}^{\leq k-3}$. For $k \leq 2$ this recovers Theorem 4.5, the general case $k \geq 3$ can be shown by a direct (but tedius) computation.

Using (4.5) we can formulate a higher order version of Corollary 4.7: If $K \in \mathcal{K}^{k}$ is a conformal Killing $k$-tensor such that the operator $\mathcal{Q}_{\lambda_{0}, \lambda_{0}}(K)$ is a conformal symmetry of $\Delta_{Y}$, then $x \mathbf{F}_{1}(K)+$ $y \mathbf{F}_{2}(K)=0$. Moreover, the same reasoning as in the proof of Theorem 4.8 yields a higher order analogue of this theorem, i.e.

$$
\mathcal{A}^{k} / \mathcal{A}^{k-1} \subseteq\left\{K \in \mathcal{K}^{k} \mid x \mathbf{F}_{1}(K)+y \mathbf{F}_{2}(K)=\mathbf{G}(\bar{K}) \text { for some } \bar{K} \in \mathcal{S}_{0}^{k-1,0}\right\} .
$$

\section{$5 \quad$ Examples in dimension 3}

In this section, we consider the space $\mathbb{R}^{3}$ endowed successively with two types of metrics: the conformal Stäckel metrics and the Di Pirro metrics.

The conformal Stäckel metrics are those for which the Hamilton-Jacobi equation

$$
\mathrm{g}^{i j}\left(\partial_{i} W\right)\left(\partial_{j} W\right)=E
$$

admits additive separation in an orthogonal coordinate system for $E=0$ (see [8] and references therein). They are conformally related to the Stäckel metrics, for which the additive separation of the Hamilton-Jacobi equation holds for all $E \in \mathbb{R}$. Moreover, the separating coordinates, called (conformal) Stäckel coordinates are characterized by two commuting (conformal) Killing 2-tensors.

Except for the Stäckel metrics, every diagonal metric on $\mathbb{R}^{3}$ admitting a diagonal Killing tensor is a Di Pirro metric g (see [28, p. 113]), whose corresponding Hamiltonian is (see e.g. [14])

$$
H=\mathrm{g}^{-1}=\frac{1}{2\left(\gamma\left(x_{1}, x_{2}\right)+c\left(x_{3}\right)\right)}\left(a\left(x_{1}, x_{2}\right) p_{1}^{2}+b\left(x_{1}, x_{2}\right) p_{2}^{2}+p_{3}^{2}\right),
$$

where $a, b, c$ and $\gamma$ are arbitrary functions and $\left(x^{i}, p_{i}\right)$ are canonical coordinates on $T^{*} \mathbb{R}^{3}$.

\subsection{An example of second order symmetry}

The Di Pirro metrics defined via equation (5.1) admit diagonal Killing tensors $K$ given by

$$
K=\frac{1}{\gamma\left(x_{1}, x_{2}\right)+c\left(x_{3}\right)}\left(c\left(x_{3}\right) a\left(x_{1}, x_{2}\right) p_{1}^{2}+c\left(x_{3}\right) b\left(x_{1}, x_{2}\right) p_{2}^{2}-\gamma\left(x_{1}, x_{2}\right) p_{3}^{2}\right) .
$$

For generic functions $a, b, c$ and $\gamma$, the vector space of Killing 2-tensors is generated by $H$ and $K$. However, for some choices of functions, this metric can admit other Killing tensors. For example, if $(r, \theta)$ denote the polar coordinates in the plane with coordinates $\left(x_{1}, x_{2}\right)$, if the functions $a, b, \gamma$ depend only on $r$ and if $a=b$, then the metric is Stäckel and admits $p_{\theta}^{2}$ as additional Killing tensor. 
Proposition 5.1. On the space $\mathbb{R}^{3}$, endowed with the metric $\mathrm{g}$ defined by (5.1), there exists a symmetry $D$ of $\Delta_{Y}$ whose principal symbol is equal to the Killing tensor $K$. In terms of the conformally related metric

$$
\hat{\mathrm{g}}:=\frac{1}{2\left(\gamma\left(x_{1}, x_{2}\right)+c\left(x_{3}\right)\right)} \mathrm{g}
$$

this symmetry is given by: $D=\mathcal{Q}_{\lambda_{0}, \lambda_{0}}(K)+\frac{1}{16}\left(3 \widehat{\operatorname{Ric}}_{a b}-\widehat{\operatorname{Scg}}_{a b}\right) K^{a b}$, i.e. by:

$$
D=\widehat{\nabla}_{a} K^{a b} \widehat{\nabla}_{b}-\frac{1}{16}\left(\widehat{\nabla}_{a} \widehat{\nabla}_{b} K^{a b}\right)-\frac{1}{8} \widehat{\operatorname{Ric}}_{a b} K^{a b},
$$

where $\widehat{\nabla}, \widehat{\operatorname{Ric}}$ and $\widehat{\mathrm{Sc}}$ represent respectively the Levi-Civita connection, the Ricci tensor and the scalar curvature associated with the metric $\hat{\mathrm{g}}$.

Proof. We use Theorem 4.11. In order to compute the obstruction $\operatorname{Obs}(K)^{b}$, we used a Mathematica package called "Riemannian Geometry and Tensor Calculus", by Bonanos [6].

This obstruction turns out to be an exact one-form equal to $d\left(-\frac{1}{8}\left(3 \widehat{\operatorname{Ric}}_{a b}-\widehat{\mathrm{Sc}}_{a b}\right) K^{a b}\right)$. The first expression of the symmetry $D$ follows, the second one is deduced from (4.4), giving $\mathcal{Q}_{\lambda_{0}, \lambda_{0}}(K)$.

\subsection{An example of obstructions to symmetries}

If written in conformal Stäckel coordinates, the conformal Stäckel metrics g on $\mathbb{R}^{3}$ admit four possible normal forms, depending on the numbers of ignorable coordinates (see [8]). A coordinate $x$ is ignorable if $\partial_{x}$ is a conformal Killing vector field of the metric.

Thus, if $x_{1}$ is an ignorable coordinate, the conformal Stäckel metrics g read as

$$
\mathrm{g}=Q\left(\left(d x_{1}\right)^{2}+\left(u\left(x_{2}\right)+v\left(x_{3}\right)\right)\left(\left(d x_{2}\right)^{2}+\left(d x_{3}\right)^{2}\right)\right)
$$

where $Q \in \mathcal{C}^{\infty}\left(\mathbb{R}^{3}\right)$ is the conformal factor and where $u$ and $v$ are functions depending respectively on the coordinates $x_{2}$ and $x_{3}$. Such metrics admit $\partial_{x_{1}}$ as conformal Killing vector field and

$$
K=\left(u\left(x_{2}\right)+v\left(x_{3}\right)\right)^{-1}\left(v\left(x_{3}\right) p_{2}^{2}-u\left(x_{2}\right) p_{3}^{2}\right)
$$

as conformal Killing 2-tensor.

Proposition 5.2. On $\mathbb{R}^{3}$, there exist metrics $\mathrm{g}$ as in (5.2) whose conformal Laplacian $\Delta_{Y}$ admits no conformal symmetry with principal symbol $K$.

Proof. Indeed, the obstruction associated with $K, \operatorname{Obs}(K)^{b}$, is generally not closed. Thanks to the Mathematica package "Riemannian Geometry and Tensor Calculus", by Bonanos [6], we can actually compute that

$$
d \mathbf{O b s}(K)^{b}=-\frac{1}{4}\left(\partial_{x_{2}}^{2}+\partial_{x_{3}}^{2}\right) \partial_{x_{2}} \partial_{x_{3}} \log \left(u\left(x_{2}\right)+v\left(x_{3}\right)\right) d x_{2} \wedge d x_{3}
$$

where the symbol' denotes the derivatives with respect to the coordinates $x_{2}$ and $x_{3}$. This expression does not vanish e.g. for the functions $u\left(x_{2}\right)=x_{2}$ and $v\left(x_{3}\right)=x_{3}$.

We conclude then using Theorem 4.8. 
An example of a metric of the form (5.2) is provided by the Minkowski metric on $\mathbb{R}^{4}$ reduced along the Killing vector field $X=x_{3} \partial_{t}+t \partial_{x_{3}}+a\left(x_{1} \partial_{x_{2}}-x_{2} \partial_{x_{1}}\right), a \in \mathbb{R}$ (see [21]). In the time-like region of $X$ and in appropriate coordinates $(r, \phi, z)$, the reduced metric is equal to

$$
\mathrm{g}=d r^{2}+\frac{r^{2} z^{2}}{z^{2}-a^{2} r^{2}} d \phi^{2}+d z^{2}
$$

and admits $\partial_{\phi}$ as Killing vector field. Moreover, after reduction, the Killing tensor $p_{x_{1}}^{2}+p_{x_{2}}^{2}$ is equal to

$$
K=p_{r}^{2}+\frac{1}{r^{2}} p_{\phi}^{2}
$$

Notice that the metric $\mathrm{g}$ is a Stäckel metric with one ignorable coordinate. Indeed, the metric takes the form (5.2), with $Q(r, z)=\frac{r^{2} z^{2}}{z^{2}-a^{2} r^{2}}, u(r)=1 / r^{2}$ and $v(z)=-a^{2} / z^{2}$, whereas the conformal Killing tensor $K-\frac{z^{2}}{z^{2}-a^{2} r^{2}} H$ can be written as in (5.3). Here, $H=\mathrm{g}^{-1}$ is the metric Hamiltonian.

In this situation, there is no conformal symmetry of $\Delta_{Y}$ with principal symbol $K$ if $a \neq 0$. Indeed, the one-form $\operatorname{Obs}(K)^{b}$ is then non-exact, as shown by Mathematica computations

$$
d \mathbf{O b s}(K)^{b}=\frac{3}{2}\left(a+a^{3}\right)\left(\frac{1}{(z+a r)^{4}}-\frac{1}{(z-a r)^{4}}\right) d r \wedge d z
$$

Remark 5.3. Extending the metric (5.2) to $\mathbb{R}^{n}$ as

$$
\mathrm{g}=Q\left(\left(d x_{1}\right)^{2}+\left(u\left(x_{2}\right)+v\left(x_{3}\right)\right)\left(\left(d x_{2}\right)^{2}+\left(d x_{3}\right)^{2}\right)+\left(d x^{4}\right)^{2}+\cdots+\left(d x^{n}\right)^{2}\right),
$$

one can check that $K$, given in (5.3), is again a conformal Killing tensor and that the one-form $\operatorname{Obs}(K)^{b}$ is in general non-exact. Thus, there is no conformal symmetry of $\Delta_{Y}$ with principal symbol $K$.

\section{Acknowledgements}

A special thanks is due to Jonathan Kress which points out a mistake in one of the example provided in the first version of this paper. It is a pleasure to acknowledge also Christian Duval and Galliano Valent for their constant interest in this work. Josef Šilhan would like to thank Pawel Nurowski for helpful discussions.

This research has been partially funded by the Interuniversity Attraction Poles Program initiated by the Belgian Science Policy Office. J. Šilhan was supported by the grant agency of the Czech republic under the grant P201/12/G028.

\section{References}

[1] Bailey T.N., Eastwood M.G., Gover A.R., Thomas's structure bundle for conformal, projective and related structures, Rocky Mountain J. Math. 24 (1994), 1191-1217.

[2] Ballesteros Á., Enciso A., Herranz F.J., Ragnisco O., Riglioni D., Quantum mechanics on spaces of nonconstant curvature: the oscillator problem and superintegrability, Ann. Physics 326 (2011), 2053-2073, arXiv:1102.5494

[3] Benenti S., Chanu C., Rastelli G., Remarks on the connection between the additive separation of the Hamilton-Jacobi equation and the multiplicative separation of the Schrödinger equation. II. First integrals and symmetry operators, J. Math. Phys. 43 (2002), 5223-5253.

[4] Boe B.D., Collingwood D.H., A comparison theory for the structure of induced representations, J. Algebra 94 (1985), 511-545. 
[5] Boe B.D., Collingwood D.H., A comparison theory for the structure of induced representations. II, Math. Z. 190 (1985), 1-11.

[6] Bonanos S., Riemannian geometry and tensor calculus (Mathematica package), Version 3.8.5, 2012, available at http://www.inp.demokritos.gr/ sbonano/RGTC/.

[7] Boyer C.P., Kalnins E.G., Miller Jr. W., Symmetry and separation of variables for the Helmholtz and Laplace equations, Nagoya Math. J. 60 (1976), 35-80.

[8] Boyer C.P., Kalnins E.G., Miller Jr. W., R-separable coordinates for three-dimensional complex Riemannian spaces, Trans. Amer. Math. Soc. 242 (1978), 355-376.

[9] Čap A., Šilhan J., Equivariant quantizations for AHS-structures, Adv. Math. 224 (2010), 1717-1734, arXiv:0904.3278.

[10] Čap A., Slovák J., Souček V., Bernstein-Gelfand-Gelfand sequences, Ann. of Math. 154 (2001), 97-113, math.DG/0001164.

[11] Carter B., Killing tensor quantum numbers and conserved currents in curved space, Phys. Rev. D 16 (1977), 3395-3414.

[12] Duval C., Lecomte P., Ovsienko V., Conformally equivariant quantization: existence and uniqueness, Ann. Inst. Fourier (Grenoble) 49 (1999), 1999-2029, math.DG/9902032.

[13] Duval C., Ovsienko V., Conformally equivariant quantum Hamiltonians, Selecta Math. (N.S.) 7 (2001), 291-320, math.DG/9801122.

[14] Duval C., Valent G., Quantum integrability of quadratic Killing tensors, J. Math. Phys. 46 (2005), 053516, 22 pages, math-ph/0412059.

[15] Eastwood M., Higher symmetries of the Laplacian, Ann. of Math. 161 (2005), 1645-1665, hep-th/0206233.

[16] Eastwood M., Leistner T., Higher symmetries of the square of the Laplacian, in Symmetries and Overdetermined Systems of Partial Differential Equations, IMA Vol. Math. Appl., Vol. 144, Springer, New York, 2008, 319-338, math.DG/0610610.

[17] Fegan H.D., Conformally invariant first order differential operators, Quart. J. Math. Oxford (2) 27 (1976), 371-378.

[18] Gover A.R., Šilhan J., Higher symmetries of the conformal powers of the Laplacian on conformally flat manifolds, J. Math. Phys. 53 (2012), 032301, 26 pages, arXiv:0911.5265.

[19] Kalnins E.G., Miller Jr. W., Intrinsic characterisation of orthogonal $R$ separation for Laplace equations, J. Phys. A: Math. Gen. 15 (1982), 2699-2709.

[20] Kolár̆ I., Michor P.W., Slovák J., Natural operations in differential geometry, Springer-Verlag, Berlin, 1993, available at http://www.emis.de/monographs/KSM/.

[21] Kozaki H., Koike T., Ishihara H., Exactly solvable strings in Minkowski spacetime, Classical Quantum Gravity 27 (2010), 105006, 10 pages, arXiv:0907.2273.

[22] Lecomte P.B.A., Towards projectively equivariant quantization, Progr. Theoret. Phys. Suppl. 144 (2001), $125-132$.

[23] Loubon Djounga S.E., Conformally invariant quantization at order three, Lett. Math. Phys. 64 (2003), 203-212.

[24] Mathonet P., Radoux F., On natural and conformally equivariant quantizations, J. Lond. Math. Soc. 80 (2009), 256-272, arXiv:0707.1412.

[25] Mathonet P., Radoux F., Existence of natural and conformally invariant quantizations of arbitrary symbols, J. Nonlinear Math. Phys. 17 (2010), 539-556, arXiv:0811.3710.

[26] Michel J.-P., Higher symmetries of Laplacian via quantization, Ann. Inst. Fourier (Grenoble), to appear, arXiv:1107.5840.

[27] Penrose R., Rindler W., Spinors and space-time. Vol. 1. Two-spinor calculus and relativistic fields, Cambridge Monographs on Mathematical Physics, Cambridge University Press, Cambridge, 1984.

[28] Perelomov A.M., Integrable systems of classical mechanics and Lie algebras. Vol. I, Birkhäuser Verlag, Basel, 1990.

[29] Radoux F., An explicit formula for the natural and conformally invariant quantization, Lett. Math. Phys. 89 (2009), 249-263, arXiv:0902.1543.

[30] Šilhan J., Conformally invariant quantization - towards the complete classification, Differential Geom. Appl. 33 (2014), suppl., 162-176, arXiv:0903.4798.

[31] Vlasáková Z., Symmetries of CR sub-Laplacian, arXiv:1201.6219. 